Bol. Acad. peru. leng. 54. 2012 (87-138)

\title{
EL SISTEMA DE CLASIFICADORES NUMERALES DEL CHOLÓN
}

\section{LE SYSTÈME DE CLASSIFICATEURS NUMERAUX DU CHOLÓN}

\section{THE SYSTEM OF NUMERAL CLASSIFICATION IN CHOLON}

\author{
José Antonio Salas García
}

\section{Resumen:}

El objetivo de este trabajo es presentar la organización del sistema de clasificadores numerales en la lengua cholona. Luego de revisar las fuentes de estudio, determinamos, en primer lugar, el estatuto morfosintáctico de los clasificadores. En segunda instancia, cotejando la caracterización gramatical con los contextos en los que aparecen, detallamos las particularidades de cada clasificador y los agrupamos de acuerdo con categorías semánticas. Finalmente, damos cuenta de un clasificador en la lengua hibito, emparentada con el cholón, y revisamos la relación de esta lengua con la familia chibcha.

\section{Résumé:}

L'objectif de cet article est de présenter l'organisation du système de classificateurs dans la langue cholona. Après avoir examiné les sources de l'étude, on détermine le statut morphosyntaxique des classificateurs. Deuxièmement, en ayant fait la comparaison des 
contextes grammaticaux dans lesquels les classificateurs apparaissent, on détaille les spécificités de chacun et on les regroupe selon certaines catégories sémantiques. Enfin, nous présentons un classificateur dans la langue hibito, liée à la cholon, et on passe en revue la relation de cette langue avec la famille chibcha.

\section{Abstract:}

The objective of this piece of work is to present the system of numeral classification used in the language of the Cholons. First of all we have looked at the written sources for this study and then we decided on the morphosyntactic status of the terms of numeral classification. After this, by comparing the grammatical characteristics revealed by confronting them with the contexts in which they appear we managed to describe thee classifying terms and their functions in detail and place them in groups of semantic categories. Finally we noticed a classifying term in a language called Hibito which is obviously related to Cholon and then we made a critical study of a relationship between a Hibito-Cholon family and the Chibcha language.

Palabras clave: numerales, clasificadores, cholón, hibito.

Mots clés: numéraux, classificateurs, cholon, hibito.

Key words: Numbers, classifiers (words which classify numerals), Cholon, Hibito, Chibcha (languages).

Fecha de recepción:

$18 / 06 / 2012$

Fecha de aceptación:

$10 / 10 / 2012$

\section{INTRODUCCIÓN}

El sistema de clasificadores del cholón ha sido comentado en más de una ocasión. Desafortunadamente, los trabajos que se han hecho en relación con esta particularidad del sistema de numerales del cholón no han ido 
más allá de lo que expone De la Mata. Brinton (1892) tan solo identifica a los clasificadores como partículas y se abstiene de mayores comentarios. El trabajo de Rivet (1956) es francamente pobre. Lo mismo podemos decir de las observaciones de Torero (2002). Otro tanto debemos de aseverar en relación con la descripción de Muysken (2004)*. Incluso el trabajo de Alexander-Bakkerus (2005) aporta escasamente a la comprensión de los clasificadores. De una tesis de quinientas treinta y tres páginas, la autora le dedica a este tema tres caras, es decir, una hoja y media. Hay que decir que a diferencia de los anteriores autores, Alexander-Bakkerus incluye objetos categorizados por los clasificadores extraídos de los textos (los cuales no fueron formulados, directamente, como ejemplos de este tema por De la Mata), y hace un esbozo de sistematización de las clases de objetos, pero sin relacionarlas entre sí. Además, plantea la existencia de clasificadores temporales, lo cual discutiremos en la cuarta sección. En las siguientes líneas, nos aprestamos a detallar las fuentes de estudio de la lengua. Luego, describiremos el sistema de numerales para, a la postre, entrar de lleno en el asunto de los clasificadores, determinando su estatuto morfosintáctico. En la quinta sección, que es el núcleo de este trabajo, agruparemos los clasificadores en categorías semánticas, que serán el fruto del cotejo de la caracterización de De la Mata con la observación de los clasificadores en variados contextos y el conocimiento que se ha obtenido a partir del estudio de otras lenguas de clasificadores. Finalmente, presentaremos evidencias de la existencia de un clasificador en la lengua hibito que junto con el cholón formaban una familia, amén de discutir la filiación del cholón con la familia chibcha.

\section{FUENTES DE ESTUDIO}

La lengua cholona ha sido documentada a través de cinco fuentes. La principal y más antigua data de 1748 . Se trata del manuscrito del Arte de la lengua cholona de fray Pedro de la Mata. De esta obra, existe una copia que se encuentra en la British Library, la cual fue efectuada en 1772 por

\footnotetext{
* Si bien en la bibliografía citamos a Willem F. H. Adelaar y Pieter Muysken, el cuarto capítulo, donde se trata el tema de la lengua cholona, es de entera responsabilidad de Muysken. De aquí en más citaremos solo a Muysken.
} 
fray Jerónimo Clota. Del original no tenemos noticias. Además de esta copia, Brinton (1892: 30) pensaba que existía otro manuscrito anónimo de esta lengua, el cual era citado por Ludewig. Revisamos la obra de Ludewig (1858: 162) y encontramos que se trata de una confusión. Si bien este cita el texto Arte de la lengua cholona, sin mencionar a su autor, esta obra no sería otra que el mismo manuscrito que hoy está en la British Library. La fuente de Ludewig era un catálogo (editado en París, en 1842) de la biblioteca de Amedée Chaumette des Fossés, antiguo cónsul francés en el Perú. Chaumette des Fossés fue propietario del Arte de la lengua cholona, antes de que llegara a la famosa biblioteca inglesa. De tal modo que nos hallamos frente a un mismo manuscrito y no ante dos, como creía Brinton. Por lo demás, el pequeño estudio interpretativo de Brinton acerca del cholón es el único que conocemos para el siglo XIX. El manuscrito de la British Library fue editado, de modo parcial, en la Revista Inca por Julio César Tello en 1923. Lamentablemente, esta edición únicamente llega hasta el folio 92, esto es, solo desarrolla por completo el primero de los cinco libros que componen el Arte y el tratado primero acerca "del nombre" del libro segundo, destinado a las partes de la oración. Si bien se dice al final de la página 750 que la edición continuará, esto es algo que no se produjo en los números posteriores de dicha revista. En el 2007, la lingüista indonesa, radicada en Holanda, Astrid Alexander-Bakkerus edita la transcripción completa del Arte de la lengua cholona. Un hecho extraño es que el manuscrito de esta transcripción fue reseñado once años antes de su publicación (Andrade 1996). Nunca habíamos visto la reseña del manuscrito de una persona viva, pero estas curiosidades tienen los estudios. Alexander-Bakkerus ha realizado un buen trabajo en su transcripión, aun cuando hay algunos errores en la misma: $\langle t y>$ en vez de $\langle$ ey $>$, en la página $42 ;<$ sifnificar $>$ en vez de $<$ significar $>$, en la página 53; <quimeñouba > en vez de <quimeñou>, en la página 88; $<$ apilui $>$ en vez de $<$ apiliu $>$, en la página 115; se omite la preposición $<D e>$ en la sexta línea de la página 203, etc. Sin embargo, hay que decir que el error es consustancial con el proceso de copia. Estas erratas son difíciles de evitar. Los beneficios de la transcripción completa superan, largamente, estos pequeños inconvenientes, que en futuras reediciones se pueden ir puliendo. Donde sí creemos que Alexander-Bakkerus (2007: 15) comete un error significativo es cuando confunde un pequeño texto 
de cuatro líneas con un poema. Ella describe el texto como un cuarteto de cuatro versos de un idioma que desconoce. Transcribimos, tal cual lo hace la autora, las cuatro líneas con la transcripción al francés del original y la traducción al castellano hecha por Alexander-Bakkerus:

$\begin{array}{lll}\text { Sho-de-ga-chu } & \text { (la main sanglante) } & \text { : la mano sangrante } \\ \text { Seet-se-be } & \text { (le soleil du midi) } & \text { : el sol de medio día (sic) } \\ \text { Mong-shong-shan } & \text { (le cypres incliné) } & \text { : el ciprés inclinado } \\ \text { Hee-la'h-deh } & \text { (la pure fontaine) } & \text { : la fuente pura }\end{array}$

Cuando vimos los supuestos versos, simplemente recordamos nuestras clases de historia del arte. No se trata de poesía de ningún tipo. Son los títulos de algunos cuadros del pintor costumbrista norteamericano George Catlin (1796-1872). Gran parte de la obra de este artista consiste en retratos de indios norteamericanos. Los personajes retratados pertenecen a las tribus hidatsa y ponca. Ambas forman parte de la familia siouan, que es la segunda familia lingüística de Norteamérica, después de la algoquina. En estas cuatro pinturas, Catlin se vale de la técnica del óleo sobre soporte de lienzo. Fueron realizadas en 1832, con idénticas dimensiones de 29 × 24 pulgadas, esto es, $73,7 \times 60,9 \mathrm{~cm}$. Todas estas obras se encuentran en el Smithsonian American Art Museum. El texto debe de haber sido escrito por el cónsul Amedée Chaumette des Fossés en el siglo XIX, que es el siglo en el que trabajó Catlin. Por esa razón, los nombres tenían una traducción al francés, por ser la lengua materna de este diplomático. Así, este misterio queda por completo resuelto.

En nuestro trabajo, hemos utilizado una copia del manuscrito que se ubica en la British Library. Sin embargo, también nos hemos beneficiado de las versiones de la Revista Inca y de la transcripción de Alexander-Bakkerus. Ahora bien, por mor de exhaustividad ofrecemos reproducciones de los cuadros de George Catlin, a fin de satisfacer la curiosidad del lector que aún no los conozca. La forma de los nombres cambia un poco en relación con la transcripción de puño y letra que hiciera Chaumette des Fossés. Los nombres de Shoo-de-gá-cha, Mong-shóng-sha y Hee-láb-dee pertenecen a la tribu ponca, mientras que el restante de Seetsé-be-a es de la tribu hidatsa. He aquí, pues, las imágenes: 


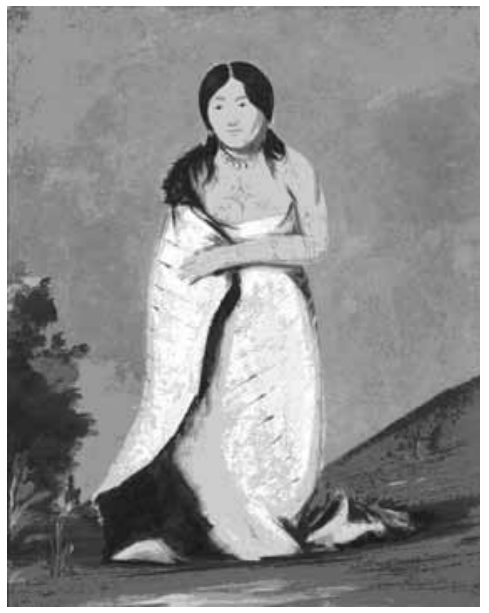

Hee-láb-dee

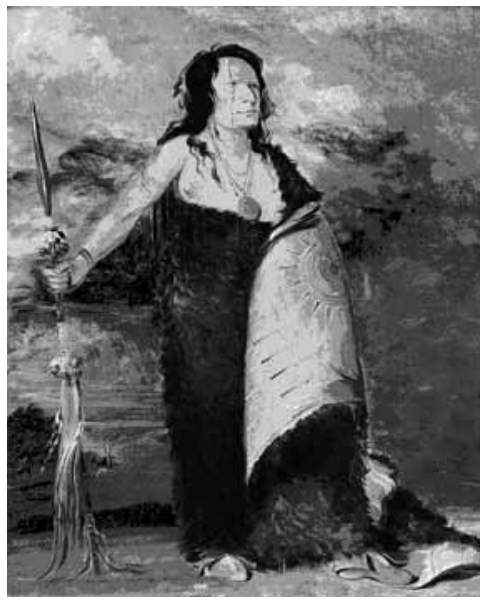

Shoo-de-gá-cha

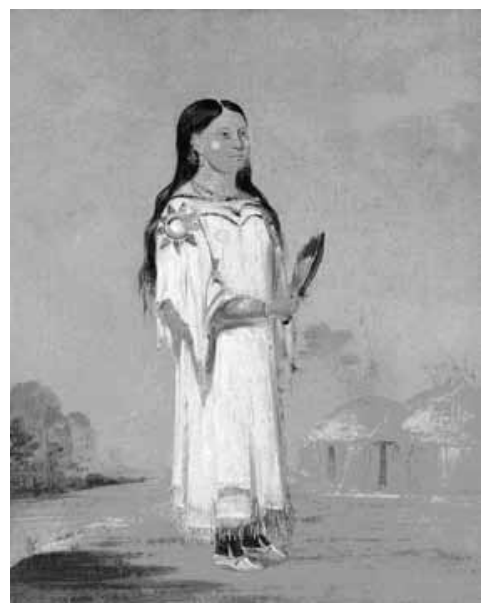

Seet-sé-be-a

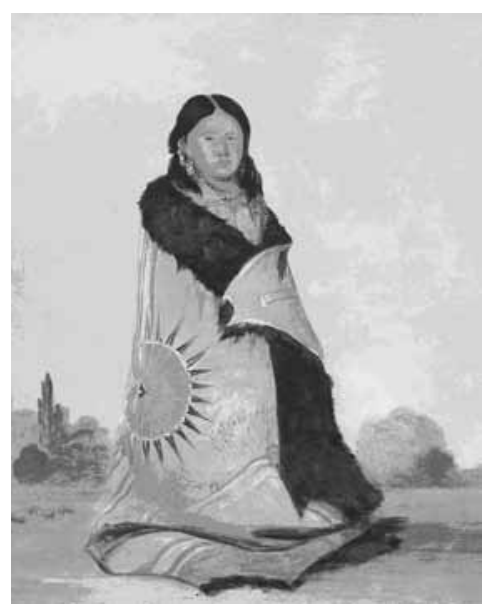

Mong-shóng-sha

En relación con el Arte de la lengua cholona, existe un estudio interpretativo inédito y sin fecha, de ochenta y cinco páginas, sobre la filiación lingüística del cholón, efectuado por John Peabody Harrington. 
Este manuscrito se encuentra en la biblioteca de la American Philosophical Society. Según hemos podido averiguar, el manuscrito cuenta con un esbozo gramatical y comparaciones con las lenguas quechua, pomo y chimariko. El título del trabajo es Affiliation of the Cholon [Andean] language.

La segunda fuente de información corresponde al Plan de 43 voces castellanas traducidas a las ocho lenguas que hablan los indios de la costa, sierra $y$ montañas del obispado de Trujillo, que se encuentra en el segundo tomo de la obra Trujillo del Perú a fines del siglo XVIII, que fuera elaborada por el entonces obispo de Trujillo Baltasar Jaime Martínez Compañón. El original de Trujillo del Perú a fines del siglo XVIII se localiza en la Biblioteca del Palacio de Madrid, toda vez que fue enviado como regalo al rey de España. Esta obra fue reeditada en 1939 por Jesús Domínguez Bordona y vuelta a reeditar entre 1978 y 1994 por la Agencia Española de Cooperación Internacional. Los cuatro primeros tomos se editaron en 1978, mientras que de 1985 a 1994 se reeditaron los cinco restantes, a los que se suman dos apéndices explicativos y la reimpresión de los de 1978. De este Plan de 43 voces existe un segundo manuscrito que se encuentra en la Biblioteca Nacional de Bogotá. Esto se debe a que Martínez Compañón fue arzobispo de San Fe de Bogotá, donde falleció en 1797. Jorge Zevallos Quiñones (1948: 119) reproduce la versión bogotana. Lamentablemente, la calidad de la reproducción no es muy buena. De las ocho lenguas que se mencionan para el obispado de Trujillo, nos interesan, particularmente, la lengua cholona y la hibito, las cuales compararemos en su momento. La lista cholón de Martínez Compañón tiene una serie de diacríticos que se distribuyen en tres posiciones: sobre vocal, sobre consonante nasal seguida de vocal y sobre consonante nasal seguida de consonante. El primer caso parece ser una marca para estipular el timbre particular de las vocales medias $e$ y 0 . El segundo tipo de diacrítico es para la nasal palatal, es decir, para la $<\tilde{n}>$. En cuanto al tercer uso del diacrítico, es para marcar una secuencia consonántica atípica para el castellano: $\langle\tilde{n} g\rangle$, cuyo valor no sería palatal, sino velar. Otro detalle a tener en cuenta es que, en ocasiones, se presentan dos palabras unidas por un signo de igualdad (=). Según hemos podido observar, este signo actúa - en un caso- para separar dos formas de un mismo concepto, 
verbigracia, al concepto 'río' se le asigna la equivalencia quõt $=$ ysoquõt. Sabemos que quõt corresponde también a 'agua' en la lista de Martínez Compañón, mientras que en De la Mata (1748: 87) se encuentra la forma xocot 'río'. No sería extraño que un pueblo mediterráneo use, eventualmente, la palabra 'agua' para designar un 'río'. Pero el signo de igualdad también se emplea para segmentar una misma palabra. A la noción de 'estrellas', Martínez Compañón le asigna la palabra que $=n a c$, que en De la Mata (1748: 88) aparece como kennà. Esto nos muestra que, en este ejemplo, el signo de igualdad (=) no separa dos palabras, sino que a lo sumo segmenta una. Debemos tener presente esto cuando usemos el Plan de 43 voces del obispo Baltasar Jaime Martínez Compañón.

La tercera fuente de estudio corresponde a las listas de palabras que proporcionó Günter Tessmann para el hibito y el cholón en su obra Die indianer Nordost-Perus, que fue editada en Hamburgo, en 1930. Ese mismo año, la obra tuvo suceso y una nueva edición vio la luz. Décadas después, en 1999, el trabajo de Tessmann fue traducido al castellano en Ecuador. La edición ecuatoriana suprime muchos de los diacríticos que aparecen en las listas de palabras y cambia sistemáticamente $\langle t \grave{s}\rangle$ por $\langle\check{c}\rangle$. Por este motivo, hemos preferido trabajar con la primera edición. Tessmann (1930: 546-547) da a conocer que a los cholones también se les conoce como tinganeses y que ellos llamaban a su lengua seeptsá. La lista correspondiente al cholón numera treinta y tres entradas, pero no da una equivalencia para tres de ellas: 'blanco', 'negro' y 'caimán'. De los treinta conceptos que restan, hay un préstamo que proviene de un arcaísmo castellano: pandtu ('plátano'); otra palabra tiene relación con la voz mochica érr ('yuca') y tres son de origen quechua: ateljwá ('gallina'), aljgó ('perro') y saš ('tapir'). Este último término es parte del compuesto 'sachavaca', cuya primera parte es quechua.

Con respecto al hibito, Tessmann trae consigo una lista con treinta y tres conceptos. Muchas de las equivalencias llevan entre paréntesis las variantes de la pronunciación, aun cuando hay casos en los que bien podría pensarse que se trata de dos lexemas distintos. En cuanto a los préstamos, la palabra palónta ('plátano') es de origen castellano. 
El término $\bar{u} d \check{s} p a$ ('gallina') provendría del quechua y la palabra compuesta satšauaa ('tapir' o 'sachavaca') es mitad quechua y mitad castellana.

La cuarta fuente corresponde a una monografía regional sobre la provincia de Huallaga, escrita por Enrique de las Casas en 1935. Esta obra trae seis palabras del cholón. Conocimos esta monografía gracias a la lectura de Benvenutto (1936: 56). No obstante, consultamos directamente el texto de De las Casas. Por error, Benvenutto solamente consigna cinco de los términos. Con ser escueto este material, presenta un hápax para la lengua cholona. He aquí la pequeña lista que comparamos con los equivalentes en De la Mata:

$\begin{array}{lll}\text { abanguez } & - & \text { abuela } \\ \text { ashol } & \text { axot } & \text { hermano } \\ \text { cot } & \text { cot } & \text { agua } \\ \text { numbul } & \text { nun }+ \text { pul } & \text { joven } \\ \text { quilacté } & \text { quillacte } & \text { vamos } \\ \text { yul } & \text { yel } & \text { sal }\end{array}$

No hemos hallado ni en el Arte de la lengua cholona ni en la lista de Martínez Compañón ni en la de Tessmann un vocablo para 'abuela'. Pese a lo minúsculo del corpus, nos encontramos ante un hápax. La primera parte de abanguez recuerda a apan ('mi madre') que aparece en De la Mata (1748: 14). El residuo guez puede analizarse gracias a la quinta fuente de estudio, en la que figura el término ges ('viejo') (Bakkerus-Alexander 2005: 527). La abuela era la 'madre vieja', lo cual tiene perfecto sentido.

La quinta fuente de estudio es una meritoria recopilación de términos efectuada por Astrid Bakkerus-Alexander (2005: 525-529). Sus informantes fueron la señora Aurelia Gutiérrez Cerquera, quien además le proporcionó algunas palabras del hibito, y el señor José Santos Chapa Ponce. La autora presenta la información léxica, dividiéndola en temas como saludos y exclamaciones, hombre y naturaleza, partes del cuerpo, animales domésticos, silvestres y acuáticos, flora, menaje y celebraciones. También brinda en la lista de Chapa Ponce el nombre de algunas enfermedades e insultos. Esta última recopilación es digna de 
encomio. De esta manera, hemos presentado el material de estudio de esta lengua. Ahora pasaremos a describir el sistema numeral del cholón.

\section{SISTEMA NUMERAL}

El sistema numeral del cholón era de base decimal y se caracterizaba por poseer clasificadores que establecían categorías, en las que se inscribían los objetos a ser contados. En lo que sigue nos ocuparemos, en primer lugar, del sistema numeral propiamente dicho y luego, en la siguiente sección, nos dedicaremos al análisis de los clasificadores. El sistema numeral es sencillo. Cuenta con bases decimales y numerales que van del uno al nueve hasta llegar a la base del sistema. El numeral correspondiente a la unidad aparece transcrito de distintas maneras:

$$
\begin{array}{ll}
\text { 1. } & <a> \\
\text { 2. } & <a l> \\
\text { 3. } & <a n> \\
\text { 4. } & <a p> \\
\text { 5. } & <a t> \\
\text { 6. } & <a x>
\end{array}
$$

Es necesario, por tanto, establecer cuál es la representación subyacente de este numeral. Las formas más fáciles de eliminar son $<a p>$ y $<a l>$, en virtud de que son predecibles por el contexto. Todos los clasificadores iniciados en $p$, verbigracia, pimoc, poc, pon, puc у puch utilizan la forma $\langle a p\rangle$. De igual manera, la forma $\langle a l\rangle$ es empleada por el clasificador liu y por la base decimal lec, que — como es obviohacen que el numeral asimile su consonante inicial. El caso de la forma $\left.<_{a}\right\rangle$ es, en realidad, una instancia más de este mismo fenómeno. Si bien parece que el numeral correspondiente a la unidad ha perdido su consonante, esto tiene una explicación simple. De la Mata (1748: 95) ofrece la forma $<$ axun $>$ que se puede analizar como a ' 1 ' y xun 'clasificador', pero el mismo autor (1748: 125) también proporciona la forma $\left\langle a x^{x} u m\right\rangle$ con una $\langle x\rangle$ añadida encima. Este hecho, además de explicar la forma $\langle a x\rangle$, nos hace pensar que también sería otro ejemplo de asimilación de la consonante del clasificador, con la diferencia de 
que en el habla rápida quizás no se pronunciaba. Lo mismo debe haber sucedido con el numeral correspondiente a la decena, que en ocasiones aparece ya como $\langle$ allec $>$, ya como $<$ alec $>$. Algo similar debe haber acontecido con la centena $\langle$ apichac $\rangle$ que bien podría analizarse como *ap ' 1 ' y pichac '100'. La forma <an> no se cuenta entre los casos de asimilación que hemos descrito. La distribución de esta forma se restringe léxicamente a dos clasificadores: zel y chup. Finalmente, la forma <at> parecería ser el resultado de otra asimilación, debido a los clasificadores tip y tuj. Sin embargo, es fácil encontrar un mayor número de ejemplos no predecibles por el contexto, a saber: los clasificadores chan, che y hil (además de los ya mencionados tip y tuj), el nombre cham 'cadena', el préstamo quechua guaranga que hace referencia al millar, el participio cotmullanco, empleado en las enumeraciones, las unidades de tiempo para mol 'mañana' y mitta 'estación', que es un préstamo quechua. De esta manera, el mayor número de contextos no predecibles, la no restricción léxica y el resultar la opción no marcada frente a los préstamos nos hace inclinar por at como la forma subyacente de la unidad. Además, esta forma nos permitiría explicar la unidad $\langle a n\rangle$ como una disimilación del momento oclusivo de las africadas de zel y chup. Es más, De la Mata (1748: 6) en un caso en el que aparece anchel, que era la forma alternativa de escribir zel, tacha la $\langle n\rangle$ y escribe una $\langle t\rangle$ encima. El numeral par ip no ofrece mayor problema. El numeral para el tres, empero, se presenta a través de tres formas:

$$
\begin{array}{ll}
\text { 1. } & <y s> \\
\text { 2. } & <y x> \\
\text { 3. } & <y c h>
\end{array}
$$

Es difícil determinar, a ciencia cierta, cuál de estas formas es la subyacente. Se puede argumentar que la consonante $s$ es la única que no sería predecible por el contexto. En apoyo de esta hipótesis se pueden presentar dobletes en los que alternan $\langle s\rangle$ y $\langle x\rangle$. Sin embargo, la distribución de $\langle y s\rangle$ se limita a un solo numeral $<y s z e l\rangle$. La forma $\langle y x\rangle$ se encuentra frente a los clasificadores: zel, ta, chup, tuj, tip, liu у а una variante de $c h e$ que se consigna como $\langle x \hat{e}\rangle$. A su vez, $\langle y x\rangle$ aparece frente a la decena lec. Finalmente, la forma $\langle y c h\rangle$ aparece frente a los 
clasificadores hil, xun y a todos los que comienzan por $p$, esto es, pimoc, poc, pon, puc, puch. Tal parece que estas formas estaban en variación libre. Así, los primeros nueve numerales de esta lengua serían:
1. at
2. ip
3. $i \check{s} \sim i \check{c}$
4. miñip
5. kiok
6. iptsok
7. kiliš
8. pak
9. okoñ

El numeral 'diez' se da por la multiplicación de la unidad por la decena: al '1' x lec '10', con el reajuste en la unidad que ya hemos señalado. El sistema numeral se incrementa sumando las unidades a la derecha de las decenas y multiplicándolas a la izquierda de las bases del sistema. De la Mata proporciona los siguientes ejemplos (De la Mata 1748: 96 y 97):

$\begin{array}{ll}\text { allec anzchel } & \text { once } \\ \text { allec ypta } & \text { doce } \\ \text { allec yxta } & \text { trece } \\ \text { alec miñip } & \text { catorce }\end{array}$

El autor añade, sin embargo, que la posposición nic 'con' se suele posponer a las unidades y nos da, a guisa de ejemplo, el numeral 'dieciséis': allec $y p^{z} c h o c$ nic (De la Mata 1748: 97). Nótese, por lo demás, cómo los clasificadores $(z e l, t a)$ de las unidades acompañan la adición de estas a la decena. Esto no sucede cuando la unidad se ubica a la izquierda de la base, multiplicando su valor, como es en la formación de los numerales que componen las decenas. He aquí los numerales en cuestión (De la Mata 1748: 96):
allec
diez
yplec
veinte 


$\begin{array}{ll}\text { yxlec } & \text { treinta } \\ \text { miñiplec } & \text { cuarenta } \\ \text { quioclec } & \text { cincuenta } \\ y p^{z} \text { choclec } & \text { sesenta } \\ \text { quilixlec } & \text { setenta } \\ \text { paclec } & \text { ochenta } \\ \text { ocoñlec } & \text { noventa }\end{array}$

Completan la lista de los cardinales, las bases decimales de las centenas y los millares, cuyo procedimiento de formación es idéntico al de las decenas arriba citadas, al multiplicar el valor de la unidad que se ubica a la izquierda de la base. El numeral correspondiente al millar es claramente un préstamo quechua. Torero (2002: 178), por su parte, llama la atención sobre el parecido de la centena pichac con el quechua *patrak, aunque sin pronunciarse al respecto (De la Mata 1748: 93):

$$
\begin{array}{ll}
\text { apižchac } & \text { cien } \\
\text { at guarnagà } & \text { mil }
\end{array}
$$

La descripción de los cardinales da paso a un sucinto repaso de lo que en castellano correspondería a los ordinales. De la Mata señala que cardinales y ordinales no tienen diferencias en el cholón. Añade, empero, una explicación que debemos cuestionar sobre la base de los mismos ejemplos que el autor proporciona. Así, De la Mata afirma, a este respecto, lo siguiente: "Hácense los ordinales usando de este participio cotmullamco que se compone del Infinitivo de Actan y de esta Partícula Mullan. Anteponiéndole el Numeral Cardinal y posponiéndole la cosa que se cuenta, según la variedad como queda advertido" (1748: 97).

El único ejemplo en apoyo de esta afirmación es at cotmullanco hil silan, al cual traduce como "el primer mandamiento". No obstante, los demás ejemplos en los que encontramos una traducción castellana con numerales ordinales no muestran ninguna diferencia con los cardinales y prescinden por completo de la partícula cotmullanco (De la Mata 1748: 97 y 242): 

at bil silan
yp bil silan
primer mandamiento
ych bil silan
segundo
tercero
miñip bilte uacho

Al parecer el uso de la partícula cotmullanco era opcional. Esto lo podemos inferir no solamente de los ejemplos arriba citados, sino de la diferencia en la distribución de la palabra axman 'primero', la cual en un caso lleva cotmullanco; y en otro, lo omite por completo (De la Mata 1748: 92 y 97$)$ :

$\begin{array}{ll}\text { Axman cotmullanco } & \text { El que está primero } \\ \text { Axman millango mectan miva } & \text { Tú tienes obligación de ir primero }\end{array}$

El término cotmullanco permitía hacer perífrasis que eran opcionales. Todo indica que no había ninguna modificación morfológica entre los numerales en el momento de expresar cantidades u órdenes. Otro tipo de numeral que De la Mata intenta describir son los llamados distributivos, los cuales se expresan en castellano a través de perífrasis, a diferencia del latín, en el que existían realizaciones morfológicas particulares (singuli, bini terni, etc.) que fueron señaladas por el propio Pedro de la Mata. En cholón, básicamente, se trata de los números cardinales (salvo la unidad que provenía de la voz yel ['solo']) dispuestos en una construcción de orden fijo, en la que se sufijaba la sílaba co a los acabados en vocal o se geminaba la consonante final de los numerales junto con una $o$ final (1748: 98):

$\begin{array}{ll}\text { yl-lo } & \text { de uno en uno } \\ \text { yptaco iptaco } & \text { de dos en dos } \\ \text { yxtaco yxtaco } & \text { de tres en tres } \\ \text { miñippô miñoppô } & \text { de cuatro en cuatro } \\ \text { quiocco quiocco } & \text { de cinco en cinco } \\ \text { ypchocco ipchoco } & \text { de seis en seis } \\ \text { quilixio quilixio } & \text { de siete en siete } \\ \text { pacco pacco } & \text { de ocho en ocho } \\ \text { ocoñ } 0 \text { ocoñ } & \text { de nueve en nueve }\end{array}$




\section{alleccò al'ecò de diez en diez

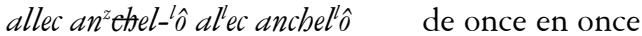

De la Mata (1748: 99) afirma que a los numerales acabados en consonante se les añade simplemente una 0 . No obstante, los mismos datos que él aporta contradicen su descripción. Nótese, por otra parte, cómo los numerales 'dos' y 'tres' llevan sufijado el clasificador ta para referirse a los humanos. De la Mata debe de haber tenido en cuenta una enumeración de humanos, al formular el ejemplo. Después de todo, eran la forma no marcada del sistema, como lo veremos en breve. El siguiente ejemplo muestra cómo se está pensando en humanos al formular la pregunta y, por tanto, en la segunda respuesta se encuentra nuevamente el clasificador para humanos (De la Mata 1748: 125):

$\begin{array}{ll}A^{n} \text { na mec iel-lom illactan? } & \text { ¿De cuántos en cuántos irán? } \\ \text { Yel-lo iel-lo, yel-loll iel-loll, } & \\ \text { yel-tell iel-tell, yel-pat iel-pat } & \\ \text { yel patall iel patall } & \text { Cada uno de por sí } \\ \text { Yptaco iptaco } & \text { De dos en dos }\end{array}$

Con todo, esta construcción de orden fijo, en la que se duplican los elementos, debe de haber sido bastante empleada, lo cual puede observarse en las siguientes frases (De la Mata 1748: 125 y 213) que también la ilustran:
Mamonte mamonte
Uno tras otro
At molle at molle
De día en día, de mañana en mañana

Nótese que en estos casos no se añade la sílaba co ni la vocal $o$, como en la construcción que permitía la expresión distributiva. De esta manera, contamos con una idea de lo que eran los numerales en el cholón. Ahora, veremos cómo interactuaban los numerales con los clasificadores.

\section{MORFOSINTAXIS DE LOS CLASIFICADORES}

En cuanto a los clasificadores del cholón, existe un consenso para considerarlos como morfemas. Brinton (1892: 33) se refiere a los clasificadores 
como partículas (particles) que indican la naturaleza de los objetos contados y cuando presenta la lista de los numerales (formas absolutas) lo hace en los siguientes términos: "The absolute forms, without these suffixes (...)". Por su parte, Rivet (1956: 179) los denomina "sufijos clasificatorios" (suffixes classificatoires). Torero (2002: 177) tendría una idea similar, pues estima que los "números dígitos requieren de la adjunción de morfemas clasificatorios (...)". En buena cuenta, se tratarían de sufijos. Para Muysken (2004: 470) serían morfemas mayormente monosilábicos. Este autor, empero, mostraría otra visión en relación con los anteriores, pues para él los numerales serían prefijos: "The numerals are prefixed to generally monosyllabic morphemes particular to a semantic class". Lo que llama la atención de esta propuesta es que siendo el numeral un hipotético prefijo se fusione con un morfema. Desde la década del setenta del siglo XX, el estudio de la morfología basada en el morfema fue sustituido por el examen de la morfología basada en el lexema. La base a la que se une un prefijo no puede ser un sufijo. Debe ser un lexema. Pese a que el trabajo de Muysken era una interpretación de los avances de investigación de Bakkerus-Alexander, esta autora tiene una opinión más cercana a la de Britton, Rivet y Torero. En efecto, Bakkerus-Alexander (2005: 179) asevera lo siguiente: "Cholón has morphemes which can be suffixed to numerals [...] and to the interrogative stem ana". Nuestra propuesta, a su turno, discrepa de todas las opiniones anteriores. Los clasificadores no serían ni sufijos ni ningún otro tipo de morfemas, sino unidades léxicas plenas. ¿Cuáles serían los indicios para afirmar que los clasificadores son lexemas y no sufijos? Presentamos los argumentos a continuación.

El primer argumento es, precisamente, la identificación de los clasificadores con lexemas en el mismo cholón. Nótese que en ningún caso hay una simplificación en la forma de las palabras. Cosa que acontece en la gramaticalización de sufijos de origen léxico. Torero (2002: 178) y Bakkerus-Alexander (2005: 181) también identifican el origen léxico de algunos de los clasificadores. He aquí la lista de algunos lexemas que dan origen a los clasificadores:

$$
\begin{array}{ll}
\text { cham 'cadena' < *cuerda } & \text { (De la Mata 1748: 199) } \\
\text { che } \sim \text { chê 'huevo' o 'grano' } & \text { (De la Mata 1748: } 104 \text { y 122) }
\end{array}
$$




$\begin{array}{lll}\text { chup } & \text { 'vientre' } & \text { (De la Mata 1748: 131) } \\ \text { bil } & \text { 'palabra' } & \text { (De la Mata 1748: 97) } \\ \text { liu } & \text { 'libro', 'escrito' o 'pintura' } & \text { (De la Mata 1748: 181) } \\ \text { poc } & \text { 'vez' } & \text { (De la Mata 1748: 95) } \\ \text { puch } & \text { 'entero' } & \text { (De la Mata 1748: 104) } \\ \text { ta } & \text { 'piedra' } & \text { (De la Mata 1748: 173) } \\ \text { zel } & \text { 'pie' o 'pierna' } & \text { (De la Mata 1748: 152 y 169) }\end{array}$

El segundo argumento para no considerar los clasificadores como sufijos es que una raíz léxica y su sufijo forman una isla. Por tanto, no se podría insertar un ítem léxico entre el numeral y el hipotético sufijo clasificador. El siguiente ejemplo nos prueba que sí se podía (De la Mata 1748: 97):

at cotmullanco bil silan el primer mandamiento

Entre el numeral at y el clasificador hil se inserta clarísimamente el ítem cotmullanco. La distribución de los clasificadores indica, además, que solían aparecer ante la palabra de pregunta cuantitativa anna. En este contexto, también se produce la violación de la supuesta condición de isla, pues se inserta el elemento léxico mec entre lo que sería la raíz y su sufijo. Por tanto, debemos concluir que la palabra de pregunta y el clasificador no formaban una isla. Simplemente, el clasificador no era un sufijo. El siguiente diálogo presta el servicio de probarlo (De la Mata 1748: 124):

\section{$A n^{n}$ a mec pucam meng̃lejtan? ¿Cuántos bocados tragarás? $A^{p}$ puc Un bocado}

Entre anna y puc se inserta mec. En consecuencia, la palabra de pregunta y el clasificador no mantenían una relación morfológica, sino sintáctica. Bakkerus-Alexander (2005: 180) propone que los clasificadores eran obligatorios para los numerales 'uno', 'dos', 'tres'. Seguramente, basaba esta afirmación en que para la cuantificación de humanos se podía prescindir de los clasificadores a partir del número 
cuatro. Presentamos los primeros seis numerales que cuantifican al ser humano de la siguiente manera (De la Mata 1748: 94):

$\begin{array}{llll}\text { anchel } & \text { 'uno' } & \text { miñip } & \text { 'cuatro' } \\ \text { ypta } & \text { 'dos' } & \text { quioc } & \text { 'cinco' } \\ \text { yxta } & \text { 'tres' } & y p^{z} c h o c & \text { 'seis' }\end{array}$

La primera columna emplea un clasificador y a partir del primer numeral de la segunda columna en adelante se prescindía de los clasificadores para contar humanos. Quizás el uso de zel en otro paradigma dé la idea de rigidez en el sistema. Por nuestra parte, creemos más bien que es un síntoma de flexibilidad. Esto se verá cuando comparemos el cholón con el hibito — otro miembro de la misma familia- que hacía uso, para la unidad, del clasificador originado en el concepto 'piedra'. Esta aparente rigidez de zel haría a Bakkerus-Alexander postular la forma anzel como un pronombre indefinido, lo cual discutiremos en su momento. El convencimiento de la obligatoriedad de los clasificadores para los tres primeros numerales motiva el análisis de algunos vocablos temporales como clasificadores por parte de esta autora. Los siguientes ejemplos muestran que los clasificadores no eran obligatorios en todos los contextos. Nótese el contraste entre el uso de hil, como nombre y como clasificador (De la Mata 1748: 97):
at bil
'una palabra'
at bil silan
'el primer mandamiento'

En el primer caso, hil es empleado como nombre; y, en el segundo, como clasificador. Por tanto, los primeros numerales sí podían aparecer sin el clasificador. Del mismo modo, podemos presentar otro indicio. Para ello, planteamos el siguiente par de ejemplos. En la primera frase tenemos el nombre cham ('cadena') sin cuantificar. Ahora, en la siguiente oración se muestra cómo el mismo nombre cuantificado por at ('uno') prescinde de cualquier clasificador (De la Mata 1748: 199):
Puillet'tzê cham
Cadena de oro
Puillethzê at cham acotan
Tengo una cadena de oro 
Al igual que hil, cham es un nombre que, a su vez, es un clasificador. La diferencia entre la frase sin cuantificar y la oración con la misma frase cuantificada nos comprueba que el uso de cham es el de un nombre propiamente dicho. En efecto, no parece que estemos frente al uso como clasificador, sino ante un uso plenamente nominal. ¿Podríamos colegir, entonces, que si se empleaba la palabra que daba origen al clasificador, no era necesario repetir el clasificador? Un contraejemplo muestra que la respuesta a la anterior pregunta es negativa (De la Mata 1748: 152):

\section{Ypethel michel nic ip tip minen nic}

Con tus dos (pierna) pies, con tus dos (pedazo) manos

Las formas zel y chel ( $<{ }^{z}$ thel michel) son variantes del clasificador y del nombre. Comprobamos así que un clasificador podía seleccionar, como un repetidor, al mismo nombre que le daba origen. Por tanto, hay una clara división entre los usos nominales y clasificatorios de hil y cham. Así, el uso nominal de estas palabras prueba que los clasificadores no eran obligatorios para los numerales 'uno', 'dos' y 'tres'. Los datos nos muestran que existían usos opcionales. Como vimos más arriba, otro contexto en el que se solía constatar los clasificadores era tras la palabra de pregunta cuantitativa ana. Empero, hay evidencias de que se podían formular, perfectamente, interrogantes cuantitativas, prescindiendo de los clasificadores (De la Mata 1748: 122, 123 y 125):

$\begin{array}{ll}\text { Ana mecam jayu chectan? } & \text { ¿Cuántos hombres son o están? } \\ \text { Ana mecam illactan? } & \text { ¿Cuántos irán? } \\ \text { Ana mec xiptetam? } & \text { ¿Cuántas anonas? } \\ \text { Ana mec ochauam? } & \text { ¿Cuántos caimitos? } \\ \text { Ana mec atellpa chem? } & \text { ¿Cuántos huevos de gallina? } \\ \text { Anna llom? } & \text { ¿Cuántas quebradas? } \\ \text { Anna xam? } & \text { ¿Cuántos pares? }\end{array}$

En tal contexto, tampoco se podría hablar de obligatoriedad en el uso del clasificador. A su vez, hay que aclarar algunas ideas con relación a las bases del sistema (decenas, centenas, millares) que no utilizan clasificadores. Las bases ocupan el lugar del clasificador, pero no clasifican 
ningún objeto. Es más, su única semántica es de tipo cuantitativo y representa tan solo una de las unidades decimales. En esta sucesión los numerales para 'uno', 'dos' y 'tres' no requerían de un clasificador antes de la decena u otras bases del sistema (De la Mata 1748: 96):

$\begin{array}{ll}\text { allec } & \text { diez } \\ \text { yplec } & \text { veinte } \\ \text { yxlec } & \text { treinta } \\ \text { miñiplec } & \text { cuarenta } \\ \text { quioclec } & \text { cincuenta }\end{array}$

Ciertos nombres contables que cuantifican entidades no contables prescinden de los clasificadores. Véase en el siguiente ejemplo cómo la palabra libra también carece de clasificador (De la Mata 1748: 6) al ser una expresión cuantitativa, una unidad de medición:

\section{Allec libra cuca pat atch ochoc amzi}

Con diez libras de coca compré mis calzones

La 'coca' es una entidad no contable. Para ser cuantificada requiere de una unidad, a fin de que esto se dé. Vemos así un nuevo caso en el que el clasificador no era requerido. Con todo, sí podemos reconocer una preferencia de los primeros numerales por emplear los clasificadores, pero no su absoluta obligatoriedad ni su carácter morfológico en tanto pretendidos sufijos. Los nombres de tiempo, a su vez, omiten los clasificadores, pero no porque ellos sean tales. Después de todo, ¿qué cosa diferente de sí mismos categorizan? Son unidades de tiempo que carecen de la semántica propia del clasificador, aun cuando puedan aparecer en contextos similares. Los nombres de tiempo, sin duda, funcionan como expresiones cuantitativas, pero no toda expresión cuantitativa es un clasificador, ya que estos categorizan clases de objetos que los diferencian de los nombres de los que toman su origen.

Una manera de probar que las expresiones temporales no son clasificadores es comprobando que la distribución de dichas expresiones temporales es diferente a la de los clasificadores. En las preguntas 
formuladas con un clasificador, la respuesta se da con el mismo clasificador (De la Mata 1748: 124 y 125).

Ana mec cham ${ }^{g a}$ veyiâ mumchan? ¿Cuántos haces has puesto de leña? Achcham Uno

Anna mec xumgam mimmollayha? ¿Cuántos montones habéis juntado? $A x^{x} u m$ Uno

En ambos casos, las respuestas asumen el mismo clasificador de la pregunta. Los dos ejemplos anteriores no son los únicos casos en los que se da este comportamiento de los clasificadores. Tan solo son una muestra de ello. A lo largo del artículo, aparecerá una mayor cantidad de ejemplos. Ahora veamos cómo son las respuestas a las interrogantes que portan expresiones temporales (De la Mata 1748: 198 y 199):

$\begin{array}{ll}\text { Ana mol patlege milluan? } & \text { ¿Por cuántos días te vas? } \\ \text { Yp semana patle } & \text { Por dos semanas } \\ \text { Ych pel patle } & \text { Por tres meses o lunas } \\ A^{p} \text { piliu patle } & \text { Por un año }\end{array}$

$\mathrm{Al}$ preguntarse por mol ('día'), se contesta con el préstamo semana o con pel ('mes') o con piliu ('año'). Comprobamos así que las expresiones temporales se comportan de forma distinta a como lo hacen los clasificadores, pues no son tales. Cuando se pregunta por un clasificador, se contesta con el mismo clasificador y no con otro diferente. Las expresiones temporales son magnitudes del tiempo, pero no categorizan clases de objetos. Aikhenvald (2003: 116) distingue entre clasificadores y expresiones cuantitativas, pues las expresiones cuantitativas mantienen su significado léxico. Confundir las expresiones temporales con los clasificadores es, mutatis mutandi, como confundir el verso de un poema con el título de un cuadro. He aquí más ejemplos de este tipo de expresiones temporales que nos regala De la Mata (1748: 199 y 213):

Ana pel patle, miloc-lobtan? ¿En cuántos meses lo acabarás de hacer? Appel patle En un mes 


$\begin{array}{ll}A^{n} n \text { a patlem? } & \text { ¿Hasta cuándo? } \\ A^{n} n \text { a peltem? } & \text { ¿En cuántos meses? } \\ A^{n} \text { na piliutem? } & \text { ¿En cuántos años? } \\ & \\ \text { At mol-le at mol-le } & \text { De día en día, de mañana en mañana } \\ \text { At mit }{ }^{t} \text { Capitep ana moltem? } & \text { Una vez } \\ \text { Cotep ip nemte patle } & \text { ¿De aquí a cuántos días? }\end{array}$

En nuestra opinión, las expresiones temporales son ejemplos de la no obligatoriedad de los clasificadores en relación con los numerales. Después de todo, tras un numeral no siempre debe haber un clasificador, pues los ejemplos que hemos presentado demuestran que existía cierta flexibilidad.

\section{CLASIFICADORES}

El sistema numeral del cholón contaba — como ya sabemos- con un conjunto de clasificadores que estipulaban las clases de objetos a ser cuantificados. En esta sección, presentaremos, primero, la información teórica basada en el trabajo de Keith Allan, para luego analizar cada uno de los clasificadores. Así, para comprender qué es un "clasificador", tomamos la definición de Allan (2001: 307):

A CLASSIFIER is a morpheme or lexeme denoting perceived or imputed characteristics of the denotatum of the associated noun, thereby classifying the referent according to one or more of eight categories - which often overlap.

Un CLASIFICADOR es un morfema o lexema que denota características percibidas o imputadas del referente del nombre asociado, de modo que lo clasifica de acuerdo con una o más de ocho categorías, que a menudo se sobreponen.

El hecho de que se mencione que las características pueden no solo ser percibidas, sino también imputadas reviste la mayor importancia, 
porque permite explicar una categoría sobre la base de creencias particulares. La investigación de Allan (1977) se inició a fines de los setenta del siglo XX con unas cincuenta lenguas. En aquel tiempo, Allan estableció solo siete categorías de clasificación. Hoy se ha incrementado esa cifra con una categoría más, correspondiente al concepto de función. Así, las ocho categorías semánticas de las que hacen uso las lenguas de clasificadores son:

1. Materia (o esencia)

2. Función

3. Forma

4. Consistencia

5. Tamaño

6. Localización

7. Disposición

8. Cantidad

Revisando los datos del cholón, podemos agrupar los clasificadores en cinco categorías, a saber, tres de forma, tres clasificadores de esencia, tres de localización, cinco de cantidad y, finalmente, una categoría residual que es una suerte de opción por defecto para cosas diversas.

\subsection{Clasificadores de forma}

Estos clasificadores determinan las clases de objetos en los que sobresale una de sus dimensiones. Allan (1977: 300) nos dice que tradicionalmente esta categoría se dividía en tres subcategorías: largo, plano y redondo. Sin embargo, los estudiosos prefieren los términos en los que se destacan una, dos o tres dimensiones. El primer clasificador, en el que predomina una dimensión, es zel, que como nombre significa 'pie' o 'pierna' (De la Mata 1748: 152 y 169-170). La longitud es una característica fácilmente percibida y en virtud de dicho atributo se debe haber empleado. Una fuente común para los clasificadores son las partes del cuerpo (Aikhenvald 2003: 442), así que este origen no debe llamar la atención. Según De la Mata (1748: 93-94), sirve para contar cuadrúpedos y cosas largas. El autor enumeró los primeros diez numerales: anzel ('uno'), ypzel ('dos'), 
yszel ('tres'), miñipzel ('cuatro'), quioczel ('cinco'), ypzoczel ('seis'), quilixzel ('siete'), paczel ('ocho'), ocoñzel ('nueve') y alleczel ('diez'). Por los datos que manejamos, en la clasificación predomina una dimensión lineal. De ahí que con este clasificador se cuenten también cabellos, huracanes, manos (seguramente por la longitud de los dedos), árboles, plátanos o pies, entre otras cosas (De la Mata 1748: 6, 7, 65, 122, 125 y 152):

$\begin{array}{ll}\text { Anchel xêe } & \text { Un cabello } \\ \text { Atchel mech } & \text { Un árbol } \\ \text { Anzel quinann ongo ocho caz } & \text { Un gran huracán } \\ \text { Anzel anente } & \text { En una de mis manos } \\ \text { An a zel mecham? } & \text { ¿Cuántos árboles? } \\ \text { Anzel palantu } & \text { Un plátano } \\ \text { Yp zèl michel nic } & \text { Con tus dos pies }\end{array}$

El último ejemplo es muy importante porque De la Mata usualmente tacha la $\langle c h\rangle$ y la sustituye por $\langle z\rangle$. Esto lo hace múltiples veces con el clasificador zel. Si se observa la palabra $<$ michel $>$, se notará que es posible analizarla como $m i$ ' $2 .{ }^{\text {a }}$ persona' + chel ('pie'); y si sustituyéramos la $\langle c h\rangle$ de $\langle$ chel $\rangle$ por la $\langle z\rangle$, nos daríamos cuenta de que el clasificador zel y el nombre chel tienen el mismo origen con distinta sintaxis y semántica. Véase como comprobación la forma de los dos primeros ejemplos de la lista. La construcción, con todo, es conocida en la literatura de clasificadores como un 'repetidor'. Sea como fuere, más adelante veremos que un mismo objeto puede ser clasificado por más de un clasificador, dependiendo del aspecto que se quiera destacar del objeto. Esto lo veremos en breve a propósito del concepto nen ('mano').

Por lo que toca al segundo clasificador, prevalecen las cosas planas, esto es, se categoriza objetos en los que sobresalen dos dimensiones. El clasificador que se emplea es chup que proviene del nombre chup ('vientre') (De la Mata 1748: 131). Nuevamente, resulta natural el empleo de una parte del cuerpo, que tiene el atributo de ser predominantemente plana, a efectos de designar una clase de objetos bidimensionales. De la Mata (1748: 94) no atinó con la generalización y tan solo hizo una larga lista de objetos en los que las dos dimensiones son predominantes, a saber, ropas, 
vestidos, hachas, machetes, peces, libros, plumas, tijeras, cuchillos, peines, zapatos o medias, al tiempo que presentaba los primeros numerales de esta clase: anchup ('uno'), ypchup ('dos'), yxchup ('tres'), miñipchup ('cuatro'), quiocchup ('cinco'), ocoñchup ('nueve') y allecchup ('diez'). El gramático, además, muestra ejemplos del clasificador en oraciones interrogativas (De la Mata1748: 123):

$\begin{array}{ll}A^{n} n a \text { chupam axua chipillte chepulli? } & \text { ¿Cuántos peces cayeron en la nasa? } \\ \text { Ana chupam menextec maton? } & \text { ¿Cuántos vestidos tienes? } \\ A^{n} \text { na chupam mihachnic mectan } & \text { ¿Cuántas hachas tienes? } \\ \text { Ana mec chupam mucuchillu nic? } & \text { ¿Cuántos cuchillos tienes? }\end{array}$

Torero (2002: 178) especulaba con que el clasificador chup era para "objetos, probablemente, de uso o aprovechamiento individuales". Bakkerus-Alexander (2005: 180) considera que los objetos que caen en esta categoría compartían la propiedad de ser portables. Ninguno de estos autores identificó el origen léxico del clasificador. Por lo que toca a la caracterización como objeto de uso individual, casi cualquier entidad cumple con tal condición, por tanto no ayuda en nada. En cuanto a lo referido al carácter portable de los objetos, esta condición también es satisfecha por cosas como frutas, que no están dentro de la categoría, además de que el hecho de ser portable no es una característica recurrente en las lenguas de clasificadores, como sí lo es la forma de los objetos.

Finalmente, el tercer clasificador de esta categoría está reservado para clases de objetos en los que predominan cosas redondas y de tres dimensiones. El clasificador che proviene del nombre che ('huevo' o 'grano') (De la Mata 1748: 104 y 122). Aikhenvald (2003: 234) da un ejemplo en baniwa, una lengua arawac, en la que el clasificador para objetos redondos también proviene del nombre 'huevo', por lo que parece natural tal designación. En efecto, De la Mata (1748: 94) enumera ocho numerales: a $a^{t} c h e$ ('uno'), ypche ('dos'), yxxê ('tres'), miñipche ('cuatro'), quiocche ('cinco'), ypzocche ('seis'), quilixche ('siete'), pacche ('ocho'); y menciona que che se usa para contar cosas redondas, aves o frutas. Las tres dimensiones explicarían la inclusión de términos como attelpa ('gallina', en tanto 'ave') o zip ('casa') (De la Mata 1748: 123 y 162): 
Ana chem zipnic puttam "â cotan? ¿Cuántas casas tiene el pueblo?

Ana chem attelpa?

Ache attellpa mullup
¿Cuántas gallinas?

Un pollo

Un caso interesante es ver cómo se clasifica un objeto artificial, claramente, venido de España. La siguiente oración hace referencia a la moneda conocida como 'real' (De la Mata 1748: 137):

Miñip che real Juan milengo cotan Tú debes dar a Juan cuatro reales

El clasificador che se emplea en virtud de la forma circular. Si bien las monedas son planas y habrían podido estar en tal categoría, se prefirió el aspecto curvo. Así, el clasificador che no solamente daría cuenta de entidades esféricas, sino también de las circulares. De esta manera, tenemos una categoría de dimensión que clasifica los objetos de acuerdo con la dimensión perceptivamente sobresaliente. Proponemos el siguiente diagrama, como un resumen de lo que contienen los clasificadores de forma:

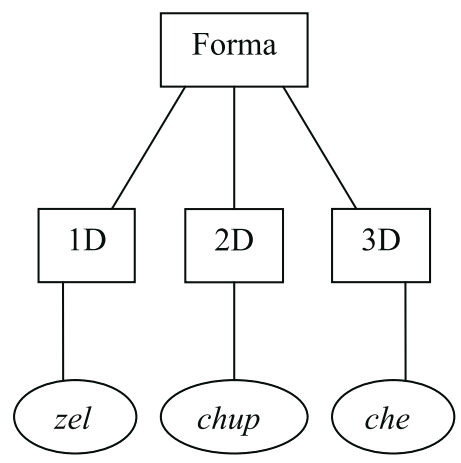

En la cabeza del diagrama, figura el ámbito semántico de la forma. Cada uno de los cuadrados que nacen de este encabezado refieren a entidades, en las prima una, dos o tres dimensiones. Finalmente, aparecen los clasificadores correspondientes. Ahora bien, la categoría de forma se hermana con la de esencia, precisamente, en un punto: la unidad del ser humano. 


\subsection{Clasificadores de esencia}

Esta categoría se divide en dos clasificadores para seres animados ( $t a$ y pon) y una clase abstracta para locuciones y enunciados (bil). Advertimos líneas arriba que la categoría de dimensión se vinculaba con la de esencia por la manera como se nombra a la unidad del ser humano. De la Mata (1748: 94) lista los numerales destinados al ser humano de la siguiente manera: anzel ('uno'), ypta ('dos'), yxta ('tres'), miñip ('cuatro'), quioc ('cinco'), ypzoc ('seis'), quilix ('siete'), pac ('ocho'), ocoñ ('nueve'), alec ('diez'). Efectivamente, es de sencilla constatación que se usa el numeral anzel para designar 'un hombre'. El ser humano cumple con la condición de poseer una orientación lineal vertical. En tal sentido, esta denominación no debe llamar a sorpresa. He aquí un ejemplo extraído de la Biblia (De la Mata 1748: 62):

\section{$A n^{z}$ thel Phariseo Un fariseo}

Con todo, la ausencia del clasificador $t a$ ('piedra') para el cholón es el resultado de la flexibilidad del sistema de clasificadores que destacan un aspecto particular. Si comparamos el cholón con el hibito, como lo haremos al final del artículo, veremos que se puede inferir también el uso de ta para el cholón. Los numerales destinados a los pares y las ternas ( $i p$ e $i x$ ) sí poseen el clasificador $t a$ ('piedra'). En este punto, es preciso explicar por qué una palabra como 'piedra' sirve para contar hombres y por qué razón los demás numerales que cuentan seres humanos carecen de clasificador. La explicación para la segunda pregunta es que la ausencia de clasificador muestra el carácter no marcado de los numerales en relación con ser humano. Esto reflejaría una visión antropocéntrica del mundo. A su vez, podría manifestar el uso tardío de esta categoría. Con respecto a la etimología del clasificador ta ('piedra'), contamos con tres hipótesis. La primera está basada en la consistencia en tanto solidez, que es por la que se inclina Bakkerus-Alexander (2005: 180). Descartamos, no obstante, esta hipótesis porque, según la investigación de 600 lenguas de clasificadores de Aikhenvald (2003: 289-290), los rasgos de consistencia no se codifican por sí mismos, sino siempre en relación con la dimensión en las lenguas de clasificadores numerales. 
La segunda hipótesis es que el concepto ta ('piedra') se haya usado para la cuantificación, lo cual no sería extraño si se considera, por ejemplo, que una palabra como 'cálculo', del castellano, proviene del latín calclus ('piedra'), en tanto elemento nemotécnico. En apoyo de esta hipótesis, hemos hallado distintos ejemplos de expresiones cuantitativas, donde figura la forma ta (De la Mata 1748: 109 y 110):

Mama ta mum puch nic hayu cotan ${ }^{\text {co }}$ Es hombre que tiene muchas riquezas Mama ta sac nic Tiene muchísimas comidas Patep mama ta checho Muchísima plata con gran exceso

Una tercera hipótesis que no es excluyente de la segunda se basa en la definición que manejamos de "clasificador". Como vimos, el clasificador "denota características percibidas o imputadas del referente del nombre asociado". La relación entre ta ("piedra') y el referente humano no solamente pudo ser percibida, sino también imputada. Curiosamente, el clasificador del mochica para contar seres humanos pong también provenía del concepto ('piedra') (Carrera 1644: 144). En el caso del mochica, la motivación era una característica imputada. Según De la Calancha (1977: 1242-1243), algunas piedras eran adoradas como deidad, porque se creía que eran los progenitores. Debido a esa creencia, tiene sentido que una piedra represente a un hombre. Lo mismo puede haber ocurrido en el cholón. Después de todo se encuentran creencias similares en el área andina. En cuanto a la distribución de este numeral, podía aparecer como un determinante de los nombres comunes o propios, como lo muestran los siguientes ejemplos (De la Mata 1748: 116):

Yxta jayu atemmê chipalli Tres hombres juntos pasaron de mañana Pedro Juannic yptapi illahuan Pedro y Juan, ambos, a dos van juntos

A su vez, los clasificadores podían determinar pronombres e incluso a ellos mismos se les podía determinar mediante sufijos, como en los ejemplos siguientes (De la Mata 1748: 213), en los que al clasificador $t a$ se le adjuntan los sufijos $-p i$ y $-(a) l l$ : 
$\begin{array}{ll}\text { Chiba yptapi } & \text { Ellos dos juntos } \\ \text { Chiba yptall } & \text { Ellos dos solos, nomás }\end{array}$

Asimismo, los numerales del cholón podían hacer las veces de pronombres, aun cuando sensu stricto no fuesen tales. Allan (1977: 286) explica que en todas las lenguas de clasificadores numerales, los clasificadores ocurren en expresiones anafóricas y deícticas. Las siguientes frases ilustran este hecho (De la Mata 1748: 97 y 117):

\section{Yptapi milobac \\ Yptapi aycha llupilan \\ Yptapi aycha llupheiton \\ Yptapi zep llupilan}

Hacedlo los dos juntos

Ambos a dos están comiendo carne Ambos a dos están comiendo carne Ambos a dos están comiendo carne

Es de primera importancia establecer el carácter deíctico de los numerales y sus clasificadores, toda vez que los clasificadores pueden designar los objetos de una clase o las clases mismas, y esto último les da un sentido similar al de los pronombres indefinidos, pues pueden referir una clase con un número indeterminado de miembros. Así, hay ejemplos en los que el numeral y su clasificador no designan a un individuo concreto, sino a la clase de individuos en abstracto (De la Mata 1748: $116,205,214)$ :

\begin{tabular}{|c|c|}
\hline$A n^{z}$ cthel sim & Es otro \\
\hline Anzthel azapte na sepeb genaqu & Queriendo coger uno, \\
\hline Anzetsel ytzpan & Coge otro \\
\hline An $n^{z}$ chel napit & Ni siquiera uno \\
\hline
\end{tabular}

No debe esto llevar a confusión. El numeral no es un pronombre indefinido. Su carácter deíctico se explica por ser una propiedad universal de los clasificadores numerales y su semántica indefinida se entiende porque el clasificador refiere tanto a objetos como a clases de objetos. Si fuese el caso que se incluyera a un numeral como pronombre indefinido, se tendría que incluir a todos los numerales en esa clase, habida cuenta de que poseen la misma propiedad, como veremos más 
adelante. En virtud de esto, disentimos de Bakkerus-Alexander (2005: 172-173) en su inclusión de anzel, como pronombre indefinido.

El segundo clasificador para seres animados es pon. La forma de este clasificador recuerda, precisamente, a la del mochica pong que, como anotamos líneas arriba, clasificaba también seres animados. Las palabras comunes que hemos encontrado entre el cholón y el mochica son más bien limitadas. El término el ('yuca') (De la Mata 1748: 9) es, sin duda, un cognado con érr ('yuca') del mochica (Bastian 1878: 172). La palabra ochô ('grande') (De la Mata 1748: 39) también parece ser cognado de $\hat{u} t z h o$ ('grande') del mochica (Carrera 1644: 15). Quizás el vocablo *setch ('cabeza') (De la Mata 1748: 101) tenga relación con lec $\mu$ ('cabeza') del mochica. Es una hipótesis provocadora el que sendos clasificadores del cholón y del mochica tuvieran un origen común. Ahora bien, a diferencia del clasificador $t a$, el clasificador pon no se restringe a los humanos, sino que se refiere a seres animados en general. Del mismo modo, otro rasgo de este clasificador es su carácter colectivo: clasifica grupos de seres animados. De la Mata (1748: 95) refiere que este clasificador designa tropas, ejércitos, compañías y manadas, haciendo la siguiente enumeración: $a^{p}$ pon ('uno'), yppon ('dos'), yx $x^{\text {sh }}$ pon ('tres'), miñoppon ('cuatro'). Otro ejemplo importante del uso de este clasificador es el siguiente diálogo, cuya importancia radica en mostrar que la distribución del clasificador también abarcaba las palabras de preguntas cuantitativas, conforme lo establece la teoría (Allan 1977: 286). He aquí el ejemplo (De la Mata 1748: 124):

\section{$A^{n} n a$ pongam quetâ vâ mipoichi?}

¿Cuántas manadas, tropas de jabalíes has visto?

\section{A pon pat illach apoichi}

Los vi ir de una tropa

Nótese, además, cómo el sentido del numeral es similar al de un pronombre indefinido, pero esto es debido a la designación de clases que hacen los clasificadores numerales. Del mismo modo, es interesante constatar cómo el clasificador pon refiere a manadas en las que se incluyen 
a los jabalíes, mas no refiere a los jabalíes mismos, que más bien habrían sido categorizados como cuadrúpedos, mediante el clasificador zel.

El tercer clasificador de esencia lo constituye el término hil ('palabra'). Obviamente, este es un clasificador tardío; resultado del encuentro entre españoles y cholones. Es, pues, un producto de la planificación lingüística por parte de los sacerdotes españoles y no podemos considerarlo como parte de la lengua cholona patrimonial. De ahí que sea un añadido en esta clase de esencia que, patrimonialmente, debe haber estado destinada a seres animados ya sea individuales o de manera colectiva. De la Mata (1748: 94) dice que sirve para contar palabras, preceptos, mandamientos y ordenanzas; y hace la siguiente enumeración: at hil ('uno'), yp hil ('dos'), ych hil ('tres'), miñip bil ('cuatro'), quioc hil ('cinco'), yp thoc bil ('seis'), quilix hil ('siete'), pac hil ('ocho'), ocoñ hil ('nueve') y allec hil ('diez'). El mismo De la Mata (1748: 97) proporciona un ejemplo en el que el vocablo bil aparece usado como nombre y no como clasificador: at hil ('una palabra'). Con todo, De la Mata también brinda ejemplos fehacientes del uso de hil como clasificador. En la lista que sigue se aprecia cómo el clasificador hil rige a la voz silan ('mandamiento') en los primeros tres ejemplos, y, en el último, se aprecia cómo al clasificador se le sufija un morfema de caso te y sufijos independientes como el topicalizador ua o la partícula adverbial cho (De la Mata 1748: 97 y 242):

$\begin{array}{ll}\text { at bil silan } & \text { primer mandamiento } \\ y p \text { bil silan } & \text { segundo } \\ \text { ych hil silan } & \text { tercero } \\ \text { Miñip bilte uacho } & \text { y el cuarto mandamiento }\end{array}$

Finalmente, De la Mata presenta una pregunta con respuesta, en donde se aprecia tanto el uso del clasificador en una construcción interrogativa de tipo cuantitativo como el empleo cardinal del numeral con clasificador (De la Mata 1748: 123):

Dios camatzin quii sil an $n$ a mec jilam?

¿Cuántos son los mandamientos de Dios? 
Allec jil-all

Solamente diez

Obsérvese que entre la palabra de pregunta anna está el cuantificador $m e c$, lo cual prueba la independencia del clasificador en tanto forma libre y no como un morfema del numeral. En suma, formulamos el siguiente diagrama que recoge, a grandes rasgos, la información que se requiere para la clasificación de esencia:

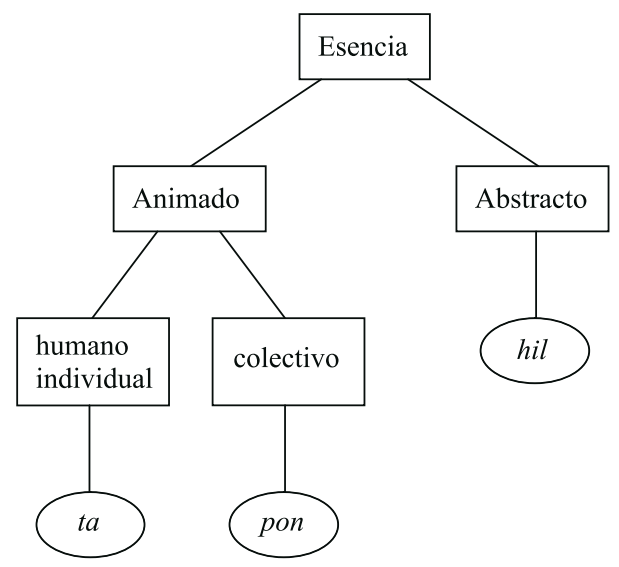

Encabeza el esquema el ámbito semántico de la esencia, del cual parten dos ramas: una para seres animados y otra para un tipo particular de entidad abstracta. Los seres animados se clasificaban, de un lado, como humanos individuales; $y$, de otro, como grupos de seres que actuaban de manera colectiva. Nótese que no hay una oposición entre humano y no humano, pues pon también contaba grupos de humanos. Tampoco era imperativo el uso de los clasificadores (de esencia) animados para todos los seres vivos. De hecho, los clasificadores de forma podían dar cuenta desde cuadrúpedos y aves hasta llegar al mismísimo ser humano. Si recordamos la definición de "clasificador", veremos que en ocasiones las categorías semánticas se superponen. 


\subsection{Clasificadores de lugar}

La siguiente categoría sirve para contabilizar lugares. Tres clasificadores la componen: puch, que se utiliza para contar chacras; pimoc, que cuenta entidades con límites establecidos; y xun, que enumera pueblos. De la Mata (1748: 95) establece que el clasificador puch sirve para contar 'chacras' y presenta los siguientes ejemplos: $a^{p} p u c h$ ('uno'), yppuch ('dos'),

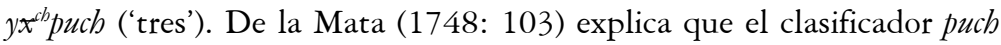
junto con los numerales significa "entero" o "cabal" y da el ejemplo allec puch ('diez enteros o cabales') y añade que con los nombres de tiempo significa 'todo'. Los siguientes ejemplos muestran el uso de puch no como clasificador, sino determinando nombres de tiempo (De la Mata 1748: 103 y 104):

A nem putch at peii A pel puch putamte at ${ }^{\tilde{n}} g u i$
Caminé todo el día

Un mes entero he estado en el pueblo

Las oraciones anteriores son importantes, porque muestran cómo el numeral $a$ ' 1 ' cuantifica nombres de tiempo, a saber, nem ('día') y pel 'mes'. En ese sentido, funcionan como unidades temporales: un día equivale a un número de horas, y un mes, a un número de días o semanas. Aikhenvald (2003: 116) hace la distinción entre clasificadores y expresiones cuantitativas, señalando que las expresiones cuantitativas mantienen plenamente su significado léxico, lo cual ya repasamos en la sección anterior. Los clasificadores, en cambio, establecen clases. El clasificador puch, que como palabra significa 'entero', clasifica 'chacras', en tanto clasificador. De la Mata (1748: 124) ofrece una pregunta con varias respuestas que permiten entender la distribución y la semántica del clasificador puch:

$\begin{array}{ll}\text { Piliu ñanmac ana } & \text { ¿Cuántas chacras hacéis cada año? } \\ \text { [pucham mihachouban? } & \\ \text { Mahall puch } & \text { Muchas } \\ \text { Cach puch } & \text { Muchas } \\ \text { Peim puch } & \text { De maní } \\ \text { Am puch } & \text { De todo género de comidas }\end{array}$

Bol. Acad. peru. leng. 54(54), 2012 
Las dos primeras respuestas muestran cómo se combinan con expresiones cuantitativas. La tercera y cuarta proporcionan la clave para entender por qué puch contaba 'chacras'. Las chacras producen en abundancia y eso es captado por la semántica de puch. Recuérdese que De la Mata nos decía que la significación de puch era 'entero' o 'cabal'. El siguiente par de oraciones (De la Mata 1748: 109 y 217) no hace más que confirmar la semántica de puch y cómo ella llevó al clasificador a la cuantificación de las chacras, sobre la base de su función en la producción de alimentos:

\section{Mamata mum puchnic bayu cotan ${ }^{c o}$}

Es hombre que tiene muchas riquezas

\section{Mahall mupuch nic, l, mamata yncha nic cotan}

Es rico, abundante en todo

El siguiente clasificador es pimoc. De la Mata (1748: 95) informa que sirve para contar cielos, entresuelos, cuartos de casa, divisiones y dobleces de ropa. Así, enumera los primeros cinco numerales de la

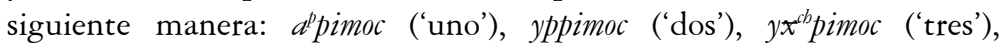
miñippimoc ('cuatro') y quiocpimoc ('cinco'). Este clasificador establecía clases de objetos en los que sus límites o bordes saltaban a primer plano. Esta noción de límites se empleaba preferentemente para entidades espaciales, pero también se aplicaba, por ejemplo, a dobleces de la ropa. Por ello, nos parece incompleta la caracterización de Bakkerus-Alexander como un simple clasificador de espacio, pues se excluye el rasgo característico de límite. De la Mata (1748: 124) consigna las siguientes preguntas en las que se utiliza el clasificador pimoc:

$A^{n}$ na mec pimocam menextec memel-lan?

¿Cuántas ropas viste?

$A^{n}$ na pimoc nic miz ${ }^{z}$ ip ${ }^{p} \hat{a}$ ?

¿Cuántos cuartos tiene su casa? 


\section{$A^{n}$ na pimocam zentà chectan?}

¿Cuántos cielos hay?

El tercer clasificador locativo es xun. De la Mata (1748: 95) nos dice que xun se emplea para contar pueblos, lugares o puestos; y enumera los primeros numerales de la siguiente manera: axun ('uno'), ypxun ('dos'), yx chxun ('tres'), miñipxun ('cuatro') y quiocxun ('cinco'). Con respecto a este clasificador, De la Mata (1748: 125) brinda dos preguntas que muestran, en primer lugar, que el clasificador selecciona nombres como putam 'pueblo' de forma explícita; y, en segundo lugar, que en las preguntas los clasificadores podrían ser prescindibles:

$A^{n}$ na mec sugam putam naime mipalliba?

¿Por cuántos pueblos habéis pasado?

Ana puttamtem mectija?

¿En cuántos pueblos estuvisteis?

Ahora bien, la clave para entender este clasificador la brinda De la Mata (1748: 125) en la traducción de la siguiente pregunta:

$A^{n}$ na mec xumgam mimmollayba?

¿Cuántos montones habéis juntado?

$A x^{x} u m$

Uno

Tal parece que xun era un clasificador que se empleaba para colecciones o agrupamientos. Así, es probable que clasificara pueblos como agrupamiento de casas, y lugares o puestos como partes de un conjunto mayor, representado precisamente por el clasificador xun. Mediante un diagrama resumimos lo visto acerca de estos clasificadores de lugar: 


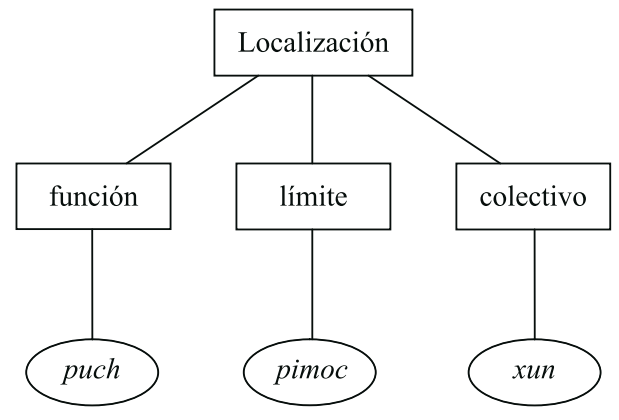

Encima de cada clasificador, está la particularidad semántica más saltante para la clasificación. Puch se empleaba para contar chacras, basándose en la abundancia de su producción. En tal sentido, una función circunscribe este espacio. La noción de límite define la clasificación del espacio para pimoc. En tanto que la agrupación de entidades, como un conjunto mayor, es explicada por el uso de xun.

\subsection{Clasificadores de cantidad}

Los clasificadores de cantidad se dividen en cinco subcategorías que, como se verá, presentan información semántica similar en ciertos aspectos con los clasificadores arriba señalados. Estos clasificadores actúan como una suerte de unidades para los objetos que contabilizan. Los clasificadores de cantidad son tip, $p u c, t u j$, chan y poc. El clasificador tip es de tipo partitivo. De la Mata (1748: 95) refiere que sirve para contar mitades o mendrugos. De este clasificador, el autor nos presenta cuatro unidades: $a^{t} t i p$ ('uno'), yptip ('dos'), yxtip ('tres') y miñiptip ('cuatro'). De la Mata (1748: 152) traduce un pasaje de la Biblia en el que se dice que es mejor entrar al cielo flaco o cojo que ser arrojado al fuego eterno, teniendo dos pies o dos manos. La última expresión cuantitativa es la siguiente:

Yp ${ }^{z}$ thel michel nic ip tip nen minen nic

Con tus dos (pierna) pies, con tus dos (pedazo) manos 
La primera parte de este ejemplo ya la habíamos citado, a propósito del clasificador para entidades largas zel, en cuya clase de objetos prima una dimensión lineal. Vimos, cuando desarrollamos dicho clasificador, que el concepto nen ('mano') podía ser cuantificado mediante zel (posiblemente porque destaca el largo de los dedos). El ejemplo de arriba, empero, nos exige una explicación satisfactoria. De obvia constatación es que una entidad como nem ('mano') podía ser clasificada por más de un clasificador. Si cada clasificador selecciona clases de cosas antes que las cosas mismas, entonces, un objeto podía ser miembro de muchas clases, dependiendo de qué aspecto del objeto se quería destacar para ser incluido en una u otra clase. Si el pasaje bíblico habla de partes del cuerpo en relación con el ser humano que ha de salvarse, entonces, es más bien natural el uso del clasificador partitivo tip. ¿Por qué no se usó también este clasificador con chel ('pie')? Se nos ocurre que las construcciones entre repetidores tenían un vínculo más fuerte entre clasificador y nombre, y - por tanto- priman sobre otros posibles clasificadores. Otra posibilidad razonable es que el autor buscó dar matices más finos a su traducción y prefirió destacar aspectos distintos en cada caso. Con todo, contamos con otro uso que pone de manifiesto el carácter partitivo de tip. En las preguntas que usualmente formula, De la Mata (1748: 123) brinda el siguiente ejemplo:

$A^{n}$ na mec tipam aycha mullupi? ¿Cuántos pedazos de carne comiste?

Esta pregunta nos hace pensar que el tamaño de las cosas categorizadas por tip es un tanto superior al del siguiente clasificador $p u c$, que se restringe al bocado que entra en la boca. De esta manera, puc restringiría su categorización no solo por el tamaño, sino también por la función alimenticia que debían de cumplir los objetos que entran en esta clase. De la Mata (1748: 95) da tres numerales de este: $a^{p} p u c$ ('uno'), $y^{p} p u c$ ('dos') y $y x^{\text {ch }}$ puc ('tres'). Una pregunta con su respuesta es lo que queda como ejemplo de uso de este clasificador (De la Mata 1748: 124):

$A n^{n}$ a mec pucam menglejtan? $A^{p} p u c$
¿Cuántos bocados tragarás?

Un bocado 
El tercer clasificador de cantidad es tuj. De la Mata (1748: 95) explica que se aplica a retazos, pedazos, nudos o junturas, y ofrece media docena de ejemplos: attuj ('uno'), yptuj ('dos'), yxtuj ('tres'), miñiptuj ('cuatro'), quioctuj ('cinco') y yphzoctuj ('seis'). Por los elementos descritos por De la Mata, consideramos que el concepto relevante para la formación de clases en este clasificador es el de límite. En ese sentido, recuerda al clasificador locativo pimoc, aunque todo parece indicar que los objetos categorizados por tuj eran, ciertamente, más pequeños. De la Mata (1748: 123) formula dos preguntas como ejemplos de uso:

$\begin{array}{ll}A^{n} \text { na tujam? } & \text { ¿Cuántos nudos o junturas? } \\ A^{n} \text { na mec tujam mimta mbac cotan? } & \text { ¿Cuántas junturas tiene tu } \\ & \text { [cuerpo? }\end{array}$

La traducción de la última pregunta nos hace pensar que un término más adecuado para juntura sería 'articulación'. Sea como fuere, el cuarto clasificador cuantitativo corresponde primigeniamente a la palabra cham *cuerda que hemos reconstruido a partir del concepto de 'cadena' (De la Mata 1748: 199). Acontece que el elemento 'cadena' era inexistente en la época prehispánica. De manera que si la palabra no es un préstamo hispano, entonces, proviene de algún referente afín del vocabulario cholón. Ese elemento similar debe de haber sido una cuerda, que además da sentido a la propuesta que haremos en relación al cuantificador. De la Mata (1748: 95) describe que este clasificador se utiliza en la cuenta de haces, manojos, atados o mazos, es decir, todas aquellas cosas que son susceptibles de agrupar con el auxilio de una cuerda; y provee, a guisa de ejemplos, tres numerales: $a^{t}$ chan ('uno'), ypchan ('dos') y $y x^{\text {th }}$ chan ('tres'). El concepto en torno al cual se organiza la categoría parece ser el de colección de elementos discretos. La cuerda se usaba para construir una unidad de objetos discretos agrupados. La noción de cuerda debe haber servido como unidad de cuantificación, que "contenía" un conjunto de elementos. De modo semejante, el clasificador pon categorizaba colecciones de seres animados. Vimos, a su turno, que el clasificador xun agrupaba partes de un conjunto mayor en el ámbito espacial. La diferencia entre chan y xun debe de haber estado dada por el tamaño más reducido de los elementos que entraban en la categoría de 
chan. Los modelos de uso (De la Mata 1748: 124) para este clasificador son los siguientes:

$\begin{array}{ll}\text { Annac chagam candela va? } & \text { ¿Cuántos mazos hay de velas? } \\ \text { Ana mec cham }{ }^{g a} \text { veyiâ mumchan? ¿Cuántas haces has puesto de } \\ & \text { [leña? } \\ \text { Achcham } & \text { Uno } \\ \text { Ypcham } & \text { Dos }\end{array}$

Nótese cómo este clasificador categoriza clases de objetos, en vez de objetos mismos. Probablemente, conceptos como candela ('vela') o vey ('leña') (Cf. ney [De la Mata 1748: 181]) habrían sido categorizados por el clasificador $z e l$, por ser largos. Sin embargo, como estos pequeños objetos estaban agrupados formando una unidad mayor, entraron en la categoría establecida por chan.

Finalmente, el último clasificador es poc y se aplica a las ocasiones en que sucede algo, o sea, a su repetición. De la Mata (1748: 95) lo traduce por 'vez' y ofrece cuatro numerales: $a^{p} p o c$ ('uno'), ypoc ('dos'), $y x^{\text {ch }} p o c$ ('tres'), miñiphoc ('cuatro'). El autor presenta una serie de ejemplos que muestran la distribución de este clasificador. El primer contexto es el más simple, como clasificador numeral (De la Mata 1748: 82):

Yppoc santo oliopat atzujiaí Segunda vez le volví a dar el santo óleo

El segundo contexto muestra al clasificador rigiendo una palabra de pregunta en una interrogante y en varias respuestas, en donde se manifiesta tanto precisa como vagamente, semejando o bien un uso numeral preciso o bien un uso indefinido (De la Mata 1748: 211):

$\begin{array}{ll}\text { An } n^{n} \text { a pocam? } & \text { ¿Cuántas veces? } \\ A^{p} \text { poc appocge } & \text { Algunas veces } \\ A^{p} \text { pocall } & \text { Una vez solamente } \\ A^{p} \text { poc } & \text { Una vez }\end{array}$

Bol. Acad. peru. leng. 54(54), 2012 
Otro contexto en el que aparece poc es rigiendo el cuantificador mec (De la Mata 1748: 211), tal como lo predice la teoría de los clasificadores numerales que mencionamos líneas arriba, pues estos aparecen en otros contextos además del entorno numeral mismo:

Ynto mec poc mige alupactan, Cuantas veces me acuerdo de ti; inco mec poc Dios muchan tanto u otras tantas, te encomiendo [amattan [a Dios

Un contexto final que está consignado en el Arte de Pedro de la Mata (1748: 124) está dado por el pequeño diálogo, en el que poc reaparece frente a una palabra de pregunta, mas en la respuesta se dan expresiones cuantitativas indefinidas, con el clasificador rigiendo elementos, evidentemente, cuantitativos.
$A^{n}$ na pocam munu tzisa milou?
Ynto mec
¿Cuántas veces has pecado?
Majall poc
Tantas veces
Mamata poc
Muchas veces
Muchas veces

Resumimos lo visto sobre este tipo de clasificadores a través del siguiente diagrama:

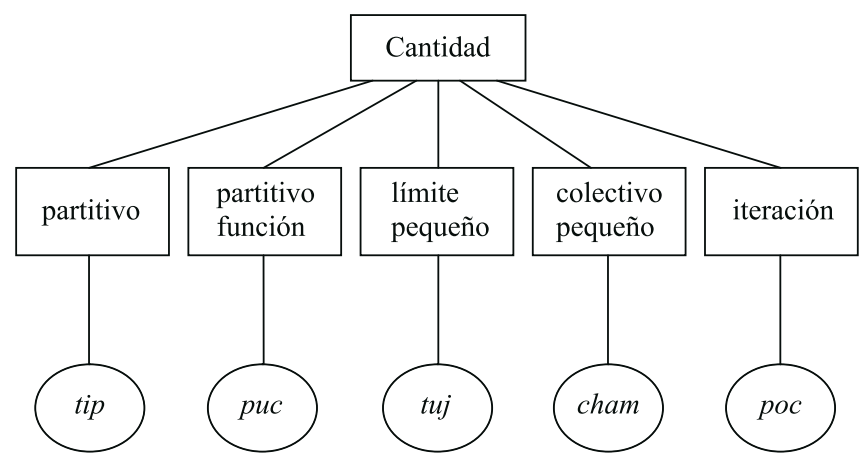

La diferencia entre el partitivo en general y el partitivo de función particular es que el segundo se restringe a la cantidad propia para ingerir 
alimentos. El tamaño parece pertinente para tuj y cham, que tienen nociones parecidas a pimoc y xun, pero para entidades más reducidas. Si comparamos los clasificadores de localización con los de cantidad, notaremos que rasgos como función alimenticia, límites y colecciones se reiteran. Esta última característica, además, está presente en el clasificador pon para seres animados. De esta manera, los resultados a los que arribamos se presentan como naturales en la lengua. Finamente, el concepto de iteración en poc parece estar más que claro.

\subsection{Categoría residual}

Esta categoría está formada por un único clasificador liu, lo cual es típico en tal tipo de categorías. Lakoff(1987: 96), Goodwin Gómez (2000: 1718) y Grinevald (2002: 220) presentan los clasificadores generales para las lenguas dyirbal, yanomami y movina, respectivamente. Allan (1977: 294), por su parte, afirma que la mayoría de lenguas de clasificadores cuenta con una clase residual. Esto prueba que la categoría residual es perfectamente común en las lenguas de clasificadores. De la Mata (1748: 95) dice que es para colores y cosas diversas, y como muestra da tres numerales: al liu ('uno'), ypliu ('dos') y ixliu ('tres'). Según De la Mata (1748: 181), liu significa 'libro', 'escrito' o 'pintura', y junto con el verbo alven forman los significados de 'pintar' o 'escribir'. Si liu significa libro o escrito, esto resulta curioso, porque esos no son elementos del mundo cholón. La traducción de liu como 'pintura' también es extraña, porque para formar el verbo 'pintar' se requiere de un auxiliar. Esto nos hace pensar que el ingreso de la palabra liu es tardío. Precisamente, el término dataría del contacto con los españoles. Si esto es así, podría tratarse de un préstamo; como en el caso en el cual liu significa 'libro'. Quizás liu provenga de un hipotético *liw-ro. Lo que habría acontecido es que la última parte de la sílaba trabada de libro se habría elidido y la consonante bilabial $b$ se habría vocalizado. A nivel conceptual, este origen tendría sentido con la diversidad que menciona De la Mata. Los libros son diversos tanto en sus formas como en sus temáticas. He aquí algunos ejemplos de uso que nos permitirán abundar en la semántica del clasificador (De la Mata 1748: 123): 
$A^{n} n a$ liu $^{\prime \prime}$ am menextec ' $\hat{a}$ ? ¿De cuántos colores es tu vestido? $\tilde{N} a p^{p} \hat{a}$ ana liuaam chectan? ¿Cuántas especies hay de papagayos? Ana mec livam chectan tua vâ? ¿Cuántas castas hay de loros?

De las dos últimas interrogantes, podemos inferir que liu establece clases antes que individuos. De ahí que se emplee en la traducción palabras como 'especie' o 'casta'. Probablemente, para contar loros o papagayos se habría empleado el clasificador che, que se utilizaba para contar aves, en tanto seres donde priman las tres dimensiones. La primera pregunta es la que suscita dudas. De acuerdo con la investigación de Allan (2001: 311), ninguna lengua de clasificadores utiliza el color como un rasgo de categorización. Este autor señala tres razones: la primera es que los colores varían con el cambio de la luz y que las sombras no son identificables perceptivamente; la segunda razón es que el color carece de forma para identificar algo; la última razón es que el color se percibe a través de un solo sentido y todas las entidades determinadas por los clasificadores son percibidas por dos o más sentidos, de modo tal que tampoco existen clasificadores de olor, de gusto o de sonidos. Por nuestra parte, creemos que el rasgo que prima en el clasificador liu es la formación de clases con una referencia inespecífica. De tal manera que clasificaba todo lo que los otros clasificadores no categorizaban, y, dentro de aquellos conceptos que quedaron de lado, quizás el color haya sido uno de ellos. De modo particular, también se debe haber empleado liu cuando no se quería destacar ningún aspecto de los miembros de una clase, como en el ejemplo de los loros, en el que no se utilizó el clasificador para aves. El siguiente diagrama es un resumen del contenido de esta categoría:

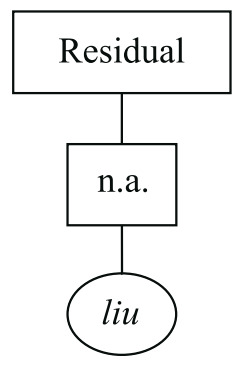


El ámbito semántico de la categoría residual está dado por la no pertenencia a ninguna de las categorías anteriores, que es lo que representa n.a. en el diagrama anterior. Si no se recurría a ninguno de los otros clasificadores y se hablaba de manera vaga o imprecisa, el clasificador liu estaba disponible. Desafortunadamente, la extinción de la lengua hace que la investigación no pueda avanzar más hasta que se hallen nuevos materiales. Este es el tipo de cosas que hay que lamentar cuando una lengua se extingue y priva al resto de la humanidad de su conocimiento. Con todo, De la Mata (1748: 216) proporciona algunos ejemplos de uso del clasificador residual:

$\begin{array}{ll}\text { Alliu } & \text { Cosa diferente, diversa } \\ \text { Alliu cotan } & \text { Es otra cosa diversa, diferente } \\ \text { Mahall liu } & \text { Muchas cosas diferentes }\end{array}$

Los dos primeros ejemplos son un indicio de que la semántica del clasificador apunta a una referencia inespecífica, además de mostrar un sentido indefinido en tanto deíctico. El último ejemplo concuerda con la semántica apuntada y nos muestra algo ya visto antes, a saber, la combinatoria que el clasificador podía establecer con expresiones cuantitativas. En efecto, los clasificadores aparecían no solo con numerales, sino también con expresiones cuantitativas que incluyen las palabras de pregunta de tal tipo.

La falta de datos en relación con esta lengua impide analizar la intuición de los propios hablantes, introducir elementos ajenos al mundo cholón para ver su clasificación, formular nuevos contextos sintácticos, investigar cómo adquieren los niños las categorías o ver qué trastornos puede haber en pacientes afásicos en relación con los clasificadores. Así de grande es el perjuicio que deja la pérdida de una lengua. Los clasificadores son una puerta abierta a los mecanismos de la mente humana. La muerte del cholón y la consiguiente desaparición de su complejo sistema numeral anulan perennemente esa posibilidad de conocimiento. Es como haber cerrado la puerta para esconder por siempre la llave. 


\section{EVIDENCIAS DE UN CLASIFICADOR EN LA LENGUA HIBITO}

En el 2004, Pieter Muysken realizó una comparación léxica para probar la filiación del cholón con el hibito. Objetivo que — según creemoslogró de manera clara. Sin embargo, su parangón pasó por alto el hecho de que en el hibito se podía encontrar uno de los clasificadores que opera también en el cholón. Esto se debe a defectos en la elección de las formas a cotejar y en la mala segmentación de las unidades. Esta es la correspondencia incorrecta que realiza aquel autor con respecto a los numerales (Muysken 2004: 461), donde M es para De la Mata y T para Tessmann:

$\begin{array}{lll}\text { cholón } & \text { hibito } & \text { glosa } \\ \text { an- } \mathrm{M} \text {, ans- T } & \text { etsí T } & \text { uno } \\ \text { ip- } \mathrm{M} \text {, hips- T } & \text { optš T } & \text { dos } \\ \text { is- } \mathrm{M} \text {, bies- T } & \text { útsi T } & \text { tres }\end{array}$

Muysken no hace la comparación entre at y etsí ('uno'), sino entre las formas an- $o$ ans- $y$ etsí, lo cual nos parece errado, pues ya habíamos establecido que la forma subyacente del numeral era at y no an. Para los numerales correspondientes a 'dos' y 'tres' no se logra establecer una correcta segmentación entre el numeral y el clasificador en el cholón de Tessmann. Por ese motivo, el cotejo no permite descubrir nada de interés. Haremos la comparación, nuevamente, desde el comienzo para mostrar lo que decimos. A la lista de cholón de Tessmann opusimos los términos de De la Mata:

$\begin{array}{lll}\text { answl } \mathrm{T} & \text { anzel } \mathrm{M} & \text { uno } \\ \text { bipswul } \mathrm{T} & \text { ypzel } \mathrm{M} & \text { dos } \\ \text { bieswúl } \mathrm{T} & \text { yszel } \mathrm{M} & \text { tres }\end{array}$

Si segmentamos los elementos comunes en ambas columnas, veremos que el equivalente del clasificador zel es swul. Tanto en el cholón como en el hibito existía la variación entre las vocales $e$ y $u$, lo que nos permite relacionar las formas de zel y swul, de modo tal que la palabra swul del cholón de Tessmann sería otra forma del clasificador zel. 
Así, podemos segmentar los numerales cholones de Tessmann de la siguiente manera: an, bip y bie. Aquí comienza la primera diferencia con Muysken. Él segmenta ans, hips y hies. Nuestra segmentación es más cercana a los datos que brinda De la Mata. Otra diferencia con relación a Muysken es que usaremos la forma no marcada de la unidad que, como vimos, es at. Así, realizamos nuevamente la comparación con el hibito:

$\begin{array}{lll}\text { cholón } & \text { hibito } & \text { glosa } \\ \text { at } \mathrm{M} & \text { etsí } \mathrm{T} & \text { uno } \\ y p \mathrm{M} \text {, hip } \mathrm{T} & \text { optš } \mathrm{T} & \text { dos } \\ \text { is } \mathrm{M}, \text { bie } \mathrm{T} & \text { uttsi } \mathrm{T} & \text { tres } \\ \text { ta } \mathrm{M}, t \mathrm{~T} & t \check{\mathrm{T}} & \text { piedra }\end{array}$

Si los numerales que ofreció Tessmann para el cholón portaban un clasificador, pensamos que los datos del hibito de este autor también portarían tal clasificador. Por ello, comparamos los numerales aislados del cholón, es decir, sin clasificador, con los datos del hibito para ver si existía un residuo común que pudiese ser identificado con algún clasificador de la lista de fray Pedro de la Mata. Así, pudimos segmentar en el hibito los numerales $e$ ' 1 ', op '2' y $\ddot{u}$ ' 3 ', quedándonos con $t s i ́, t \check{s}$ y $t s i$, respectivamente. Todas estas formas, por su similitud, deben de poseer un origen etimológico común. La forma $t \check{s}$ del numeral par es idéntica a la palabra tš 'piedra' que Muysken, correctamente, identificó como cognado del cholón ta. Ahora bien, si revisamos la lista de clasificadores, notaremos que existe un clasificador $t a$, cuyo origen es el concepto 'piedra' y que servía para contar 'humanos' (De la Mata 1748: 94). Tenemos, pues, identificado un clasificador para el hibito. Cuando revisamos la lista de los clasificadores del cholón, establecimos que la forma no marcada de los numerales era para contabilizar humanos. Al ser el concepto no marcado por excelencia, tiene sentido que los hablantes de hibito hayan mencionado, precisamente, este clasificador para indicar los numerales para 'uno', 'dos' y 'tres'. Este hecho no se hubiera podido ver si nos hubiésemos quedado con una segmentación incorrecta. La importancia de esto no solamente radica en el hallazgo léxico, sino que permite postular una estructura común entre el cholón y el hibito de tipo 
semántica y gramatical, como la de los clasificadores, fundamentando así la filiación. A su vez, nos prueba que el clasificador para 'piedra' se utilizaba para la unidad en una lengua emparentada con el cholón. Consecuentemente, también da pie para pensar que este clasificador se debe de haber empleado en algún momento en el cholón.

\section{FILIACIÓN CON LA FAMILIA CHIBCHA}

En 1949, Rivet creía poder probar el parentesco entre la familia hibito-cholón y el chibcha sobre la base de ochenta y seis cognados. Sin embargo, la comparación es, metodológicamente, deficiente. Por ejemplo, se incluía como dos cognados diferentes los lexemas pandt y palónta o palantu, que remitían a la noción de banana, que es un préstamo hispánico. Formaba parte de la comparación el término cholón para la centena, aun cuando era de origen quechua. Además se cuenta cognados diferentes para cada forma de un mismo concepto incrementando, artificialmente, el número de lexemas "comunes". Así, aparecen dos conceptos para 'fuego', 'hierba', 'luna, 'yuca', 'morir', 'padre', 'pescado', 'tierra', e incluso hasta tres conceptos para 'ave' y 'agua', por no mencionar que algunas de las comparaciones carecen de fundamento. Finalmente, se han contrastado los términos de la familia hibito-cholón con cualquier vocablo de cuarenta y cinco variedades de la familia chibcha, sin establecer relaciones entre las mismas.

No contento con este trabajo, Rivet (1956: 182) busca sustentar la relación entre el chibcha y el cholón presentando los datos de dos variedades chibchas: bribri y čiripó. El argumento es que los clasificadores de estos idiomas se parecen a los del cholón y eso refuerza el vínculo entre ambas familias. Lo que Rivet no tuvo en consideración es que las lenguas de clasificadores funcionan de manera similar, pese a no tener relación genética. Una lengua de África puede tener una clasificación semejante a una de América sin que medie vínculo directo. Que se clasifiquen cosas redondas, personas u objetos alargados no indica parentesco alguno. Las lenguas del mundo realizan categorizaciones análogas. Veamos si los clasificadores postulados por Rivet comparten sus formas con los del cholón. En la lengua bribri, para objetos redondos, frutas, piedras y 
años, Rivet (1956: 182-183) postula múltiples formas: $-k,-\underline{l},-l,-r$, pero ninguna de ellas es similar al clasificador $c h e$ de objetos redondos o donde priman tres dimensiones. De igual manera, para contar casas, hogares o tumbas familiares se emplea $-t k$, que no se parece en absoluto a los clasificadores del cholón pimoc o xun. El clasificador tb de la lengua bribri servía para cuantificar árboles, ríos, ganado, jaguares, peces, cadenas, flechas, arcos, cerbatanas. Da la impresión de que sería un clasificador para objetos donde prima una dimensión, es decir, largos. Sin embargo, no encontramos parecido entre la forma $t b$ y el clasificador zel del cholón. Para el clasificador - $t$, Rivet enumera: cestas, camisas, pantalones, dientes, manos, aves, tiempo, campos de maíz y objetos. Quizás, este clasificador funcionaba como opción por defecto, pero no es análogo con el cholón liu.

En bribri, el clasificador - $t$ cuantificaba hombres, personas y dioses. La forma de este clasificador no mantiene ningún tipo de semejanza con el clasificador $t a$ del cholón. A su vez, el clasificador del bribri -kat usado para cuantificar árboles de cacao y otros frutales no tiene equivalente en el cholón. Por último, el clasificador bribri džk para 'fardos' es completamente diferente de chan que sería el más afín en cholón.

En cuanto a la lengua čiripó, Rivet (1956: 183 y 184) brinda cuatro clasificadores: - kă para 'bananas', -ŭă para 'años', -ăbă para 'peces' y - $t$ para 'naranjas' y 'árboles frutales'. Ninguno de estos clasificadores se asemeja ni remotamente a alguno de los del cholón ni en forma ni en significado. El mismo Rivet (1956: 184) menciona, de manera inatingente, que la lengua move-valiente (del grupo guaymí) posee, para contar los días, un sufijo -era; y, para contar los meses, un prefijo mana-. Esto nada tiene que ver con el cholón. Asimismo, Rivet (1956: 184 y 185) alude a los prefijos clasificatorios del cuna: kua- (nombres variados incluyendo animales), ka- (lápices, cigarros, armas de fuego, peces), war- (personas y animales), sar- (árboles y plantas), y los nombres comunes kia- ('pieza para contar cuerdas'), or- ('pedazo para contar objetos redondos') y matta- ('hoja de papel'). Nuevamente, no existe ninguna semblanza ni de forma (los clasificadores del cholón no antecedían a los numerales) ni de contenido con respecto al cholón. Estamos seguros de que es más lo que ignoramos 
que lo que conocemos sobre los clasificadores de esta lengua cholón, pero podemos afirmar que con lo poco que sabemos, estamos en condición de descartar que los clasificadores del cholón tengan relación con los del chibcha. Finalmente, estamos de acuerdo con la interpretación de Torero (2002: 177) sobre la posible existencia de un mayor número de clasificadores en el cholón, a la luz del texto de De la Mata (1748: 93), en el que menciona que pondrá "ejemplo de las cosas más ordinarias". Por desgracia, quizás estemos condenados a no tener jamás noticia de lo más extraordinario. 


\section{BIBLIOGRAFÍA}

ADELAAR, Willem Frederik Hendrik \& Pieter CORNELIS MUYSKEN 2004 The languages of the Andes. Cambridge: Cambridge University Press.

AIKHENVALD, Alexandra Yurievna

2003 Classifiers. A Typology of Noun Categorization Devices. Oxford: Oxford University Press.

ALLAN, Keith

1977 "Classifiers". En: Language, volume 53, number 2, pp. 285-311.

$2001 \quad$ Natural Language Semantics. Oxford: Blackwell Publishers.

ANDRADE CIUDAD, Luis Florentino

1996 "Pedro de la Mata. Arte de la lengua cholona (1748), transcripción del manuscrito 24,322 de la British Library por Astrid Alexander-Bakkerus, s/f”. En: Boletín de la Academia Peruana de la Lengua, 27, pp. 171-180.

BASTIAN, Philipp Wilhelm Adolf

1878 Die Culturländer des Alten America (= Ein Jabr auf Reisen. Kreuzfahrten zum Sammelberuf auf transatlantisches Feldern der Ethnologie, erster Band). Berlin: Weidmannsche Buchhandlung.

BAKKERUS-ALEXANDER, Astrid 2005 Eighteenth-Century Cholon. Utrecht: LOT.

BENVENUTTO MURRIETA, Pedro Manuel

1936 El lenguaje peruano. Tesis para optar el grado de Doctor en Historia y Letras. Tomo I. Lima: Sanmarti y Cía. 
BRINTON, Daniel Garrison

1892 Studies in South American Native Languages form MSS. and rare printed sources. Philadelphia: MacCalla \& Company.

CARRERA DAZA, Fernando de la 1644 ARTE DE LA LENGVA YVNGA DE LOS VALLES del Obispado de Truxillo del Peru, con un Confessonario, y todas las Oraciones Christianas, traducidas en la lengua, y otras cosas. Lima: Ioseph de Contreras.

CASAS, Enrique de las

1935 Provincia de Huallaga: apuntes monográficos. Lima: Librería e Imprenta Gil.

GOODWIN GÓMEZ, Gale

2000 "Noun classifiers in ethnobotanical terminology of a Yanomami language of Brazil". En: VOORT, Hein van der \& Simon van de KERKE (eds.): Indigenous Languages of Lowland South America. (= CNWS PUBLICATIONS Vol. 90 \& Indigenous Languages of Latin America (ILLA), Vol. 1). Leiden: Research School of Asian, African and Amerindian Studies, pp. 9-24.

GRINEVALD, Collette

2002 "Nominal classification in Movina" (= Selected paper form the $50^{\text {th }}$ International Congress of Americanists in Warsaw). En: CREVELS, Mily, Simon van de KERKE, Sérgio MEIRA \& Hein van der VOORT (eds.): Current Studies on South American Languages (= CNWS PUBLICATIONS Vol. 114 \& Indigenous Languages of Latin America (ILLA), Vol. 3). Leiden: Research School of Asian, African and Amerindian Studies, pp. 215-239.

\section{LAKOFF, George P.}

1987 Women, Fire and Dangerous Things. What Categories Reveal about the Mind. Chicago and London: The University of Chicago Press. 
LUDEWIG, Hermann Ernst

1858 The Literature of American Aboriginal Languages (= Trübner's

Bibliotheca Glottica I). London: Trübner and Co.

MARTÍNEZ COMPAÑÓN, Baltasar Jaime

[1782-1790]

1985

Trujillo del Perú en el siglo XVIII. Tomos I y II. Madrid: Ediciones de Cultura Hispánica.

MATA, Pedro de la

$1748 \quad$ Arte de la Lengua Cholona. Trujillo: MS.

[1748] 1923 “Arte de la Lengua Cholona”. En: Inca, volumen I, número 3, pp. 690-750.

[1748] 2007 Arte de la Lengua Cholona. Transcripción y edición preparada por Astrid Alexander-Bakkerus con prólogo de Willem Frederik Hendrik Adelaar. Madrid: Iberoamericana, Vervuert.

RIVET, Paul Adolph

1949 "Les langues de l'ancien diocèse de Trujillo". En: Journal de la Société des Américanistes, tomo XXXVIII, pp. 1-51.

1956 "Les affixes classificatoires des noms de nombre". En: Journal de la Société des Américanistes, tomo XLV, pp. 179-187.

\section{TESSMANN, Günter}

1930

Die indianer Norost-Perus. Grundlegende Forschungen für eine systematische Kulturkunde (= Veröffentlichung der HarveyBassler-Stiftung Völkerkunde II). Hamburg: Friedersichsen, de Gruyter \& CO. M. B. H.

[1930] 1999 Los indígenas del Perú nororiental (= Publicación etnológica de la fundación Harvey-Bassler, tomo II). Traducción de Gunda Wierhake. Quito: Ediciones Abya-Yala. 


\section{TORERO FERNÁNDEZ DE CÓRDOVA, Alfredo Augusto}

2002 Idiomas de los Andes. Lingüística e historia (= Travaux de l'Institut Français d'Études Andines 162). Lima: IFEA \& Editorial Horizonte.

\section{ZEVALLOS QUIÑONES, Jorge}

1948 "Primitivas lenguas de la costa". En: Revista del Museo Nacional, tomo XVII, pp. 114-119.

\section{Correspondencia:}

José Antonio Salas García

Licenciado en Lingüística y Literatura Hispánica con mención en Lingüística por la Pontificia Universidad Católica del Perú.

Correo electrónico: jos778@hotmail.com 